\title{
capacité portante des semelles filantes
}

\author{
par
}

M. Matar

Docteur-Ingénieur

et

\author{
J. Salençon \\ Professeur \\ Laboratoire de Mécanique des Solides \\ (Ecole Polytechnique, Ecole des Mines de Paris, \\ Ecole Nationale des Ponts et Chaussées)
}

\section{Introduction}

Le dimensionnement des fondations superficielles s'effectue habituellement en se référant à deux critères : critère de tassement et critère de poinçonnement:

- par le premier on cherche à limiter la charge admissible pour la fondation par la condition qu'un certain tassement, absolu ou différentiel, ne dépasse pas la valeur jugée tolérable pour la bonne tenue de l'ouvrage concerné ; la relation chargement-tassement, nécessaire à la mise en œuvre de ce critère de dimensionnement, est obtenue soit par des calculs utilisant le plus souvent pour le sol un modèle de comportement élastique linéaire isotrope, soit par des formules reliées à des essais en place ;

- pour le deuxième critère, on s'intéresse à la "stabilité " de la fondation et on limite la charge admissible pour cette dernière à une certaine fraction de la capacité portante. $\mathrm{Ce}$ concept de capacité portante se réfère à une analyse de la fondation "à la rupture " basée sur la donnée du critère caractérisant la « résistance " du sol de la fondation.

On s'intéressera ici plus particulièrement à l'aspect capacité portante pour les fondations de type «semelle filante " chargées axialement. Toutefois certains des raisonnements mis en œuvre auront une portée plus générale et pourront être transposés sans difficulté à d'autres types de fondations et à d'autres cas de chargements.

Diverses formules d'usage courant permettent d'évaluer la capacité portante des semelles filantes à partir des paramètres caractérisant la résistance du sol, qui figurent dans les critères classiques de Coulomb (C, $\Phi$ ) ou de Tresca (C) pour les sols de fondations illimités et homogènes ( $\left.{ }^{2}\right)$.

A partir de cette estimation de la capacité portante on limite la charge admissible pour la fondation par l'application d'un coefficient de sécurité qui vise à prendre en compte les divers aspects suivants (figure 1):

1 La modélisation qui fait passer du problème pratique " dimensionner une fondation réelle pour assurer le bon fonctionnement de l'ouvrage concerné "

au problème schématique dans lequel

« la fondation est supposée de type semelle filante, infiniment rigide, soumise à un chargement uniaxial détermi-

(1) $\mathrm{C}$ et $\Phi$ sont utilisés ici comme notations génériques pour la cohésion et l'angle de frottement, sans aucune signification particulière. niste, reposant sur un sol illimité et homogène " :

2 Le fait que pour ce problème modélisé on n'obtient qu'une réponse de portée plus ou moins limitée, en ne prenant en compte, en particulier, qu'un aspect du comportement du sol : sa " résistance »:

3 L'adaptation de cette réponse partielle au problème modélisé, à la question posée par le problème pratique réel.

Remarquons qu'au niveau du point 2 , on est ramené à un pur problème de mécanique sur lequel on peut mettre en cuvre des raisonnements rigoureux permettant de donner, aux réponses obtenues, des significations précises.

Le but du travail rapporté ici est d'améliorer l'état des connaissances sur le problème modélisé indiqué ci-dessus à la fois dans le cas classique du sol de fondation illimité et homogène et dans le cas de la couche de sol d'épaisseur limitée et de cohésion linéairement variable avec la profondeur. Cela signifie que l'on fournit pour ce problème des réponses dont la signification mécanique est plus précise que celles actuellement disponibles lorsqu'elles existent : ceci s'appuie, pour le raisonnement mécanique, sur la théorie du Calcul à la rupture (cf. p.ex. Salençon, 1978) et sur les calculs nouveaux effectués par Matar (1978).

De plus, une comparaison avec des résultats d'essais réalisés en laboratoire sur modèles réduits par Tournier (1972) et Tournier et Milovic (1977) est présentée pour tenter d'étalonner, vis-à-vis de ce problème modélisé, le caractère partiel de la réponse obtenue, c'est-à-dire la signification vis-à-vis de ce problème de la capacité portante ainsi calculée: en effet la capacité portante apparait par la théorie comme la charge "extrême » pour la fondation et il convient de la situer, par l'expérience, par rapport aux charges entraînant la ruine de celle-ci ; ceci permet entre autres d'évaluer l'importance de la forme retenue pour le critère de rupture pour le sol, des déformations avant rupture, etc.

Le plan de l'article qui ne traite donc que du problème modélisé est ainsi le suivant:

le chapitre 2 est consacré à la définition de la capacité portante en montrant que le problème posé est typiquement un problème de Calcul à la rupture, et en signalant en particulier que la méthode de superposition utilisée ciassiquement en est une application directe : 


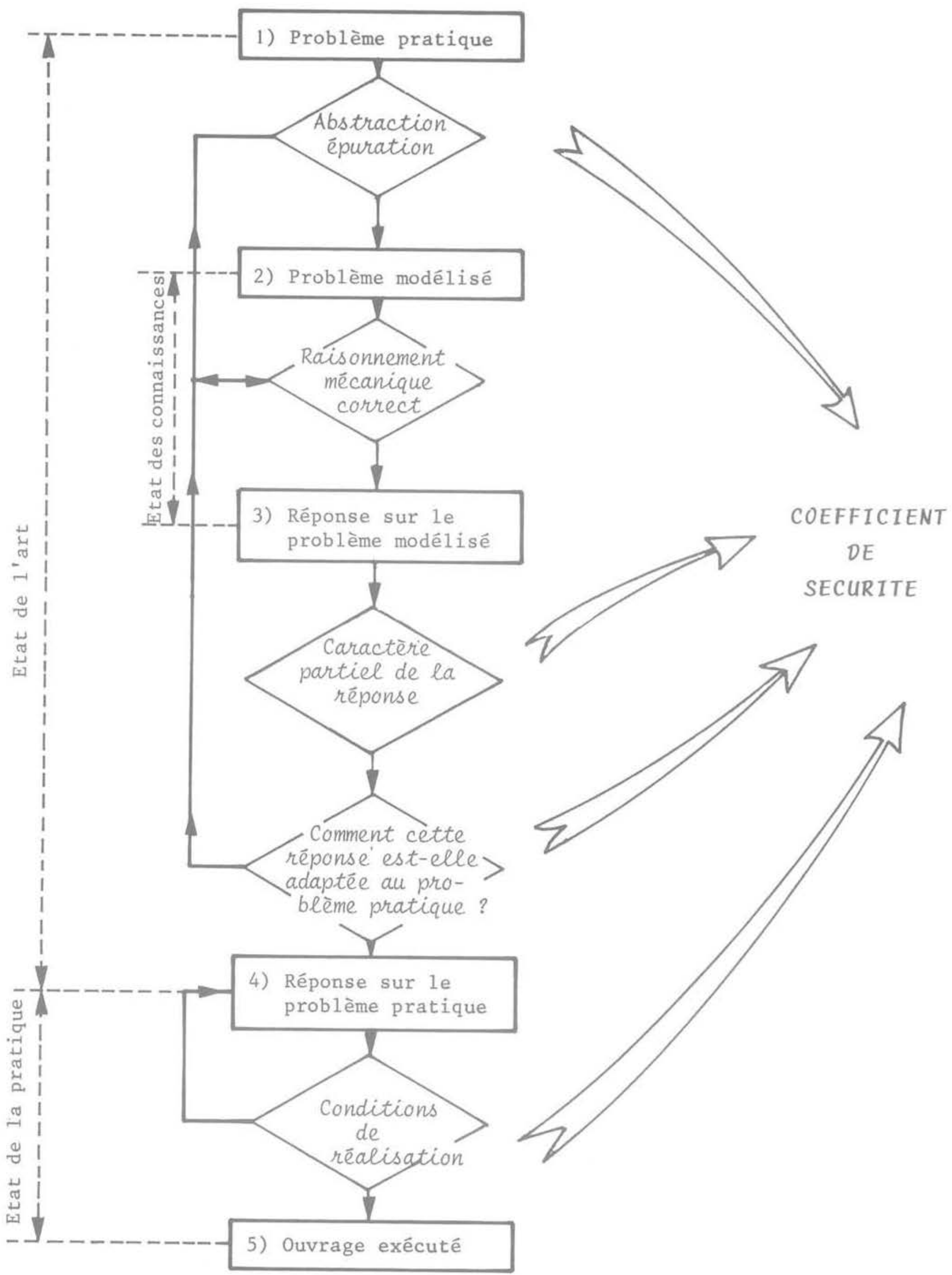

1) Dimensionner une fondation pour assurer le bon fonctionnement de l'ouvrage

2) Semelle filante, rigide; critère de rupture du sol; mode de chargement déterministe

3) Abaques pour le calcul de la capacité portante

4) Dimensionnement de la fondation

5) Fondation exécutée (lieu, conditions diverses, entreprise...)

Fig. 1 Schématisation du processus de raisonnement relatif à la capacité portante des fondations superficielles 
au chapitre 3 , on analyse le cas de la fondation sur couche de sol d'épaisseur limitée et de cohésion linéairement variable avec la profondeur et on montre qu'il est possible de lui appliquer la méthode de superposition pour obtenir une première évaluation non triviale de la capacité portante, au moyen de résultats antérieurement connus :

au chapitre 4 , une analyse globale du problème est faite qui conduit à la mise en œuvre de calculs nouveaux fournissant la valeur de la capacité portante en tenant compte des effets de couplage entre tous les paramètres du problème :

ces résultats sont présentés au chapitre 5 sous forme d'abaques dans le but d'aboutir à une utilisation pratique commode :

on en donne des exemples d'application au chapitre 6 , mettant en évidence, dans certaines circonstances, l'importance des effets de couplage négligés par les formules usuelles dans les cas classiques, et en montrant, pour le cas non-classique de la couche de sol de cohésion variable, les inconvénients, voire les dangers, que peut entraîner l'usage de certaines règles empiriques, d'ailleurs souvent assez malcommodes:

au chapitre 7 , on évoque la comparaison avec les essais sur modèles réduits.

La rédaction de cet article sera volontairement succincte sur certains aspects, en particulier en ce qui concerne les programmes de calcul mis au point et les calculs numériques effectués; le lecteur intéressé pourra se reporter essentiellement à la référence (Matar, 1978).

\section{Le concept de capacité portante}

\subsection{Les formules classiques}

Comme on l'a déjà indiqué plus haut, de nombreux auteurs ont proposé des formules pour l'évaluation de la capacité portante des fondations superficielles de type semelle filante, reposant sur un sol illimité homogène. Dans ces formules, le sol est caractérisé par son critère de rupture (cf. Salençon et Halphen, 1979) ; désignant par $\sigma$ et $\tau$ les composantes normale et tangentielle du vecteur contrainte $I$ agissant sur une facette de normale rentrante $n$ (figure 2 ), on adopte :

pour les sols purement cohérents (comportement à court terme), le critère de Tresca,

$$
|\tau| \leqslant C, \forall \underline{n}
$$

pour les autres sols, le critère de Coulomb

$$
|\tau| \leqslant C+\sigma \operatorname{tg} \Phi, \forall \underline{n} \quad(\Phi \neq 0) .
$$

Les formules proposées pour la capacité portante de la semelle filante de largeur B, chargée axialement par une force $Q$ (par unité de longueur courante), agissant sur un sol pesant de poids volumique $\gamma$, avec une surcharge latérale uniforme q (figure 3) s'appuient sur la méthode de superposition, dont on admet le caractère conservatif, et qui conduit à la décomposition de la capacité portante en trois termes sous la forme (Terzhagi, 1943):

$$
\left(\mathrm{q}_{\mathrm{u}}\right)_{\text {superp. }}=\frac{1}{2} \gamma \mathrm{BN} \mathrm{N}_{\gamma}(\Phi)+\mathrm{CN}_{\mathrm{c}}(\Phi)+\mathrm{qN}_{\mathrm{q}}(\Phi)
$$

Ainsi le calcul de la capacité portante nécessite, en s'appuyant sur la formule (2.3), la connaissance des coefficients scalaires $N_{0}(\Phi), N_{c}(\Phi)$ et $N_{G}(\Phi)$ intervenant dans les termes de surface, de cohésion et de surcharge. Les valeurs retenues pour les coefficients $N_{f}(\Phi)$ et $N_{0}(\Phi)$ sont actuellement pratiquement identiques pour tous les auteurs ; par contre des divergences apparaissent concernant $\mathrm{N}_{\gamma}(\Phi)$.

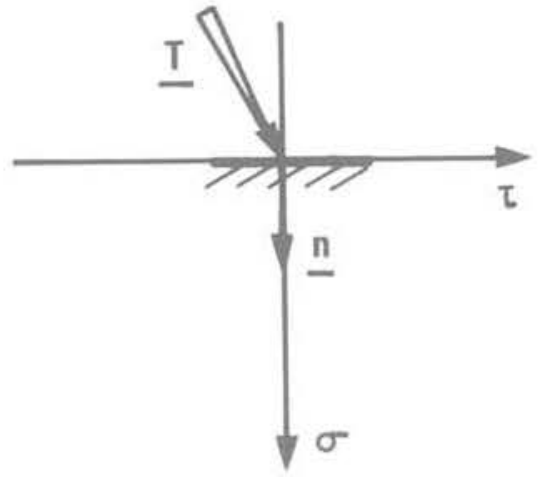

Fig. 2 Convention de signes sur les contraintes

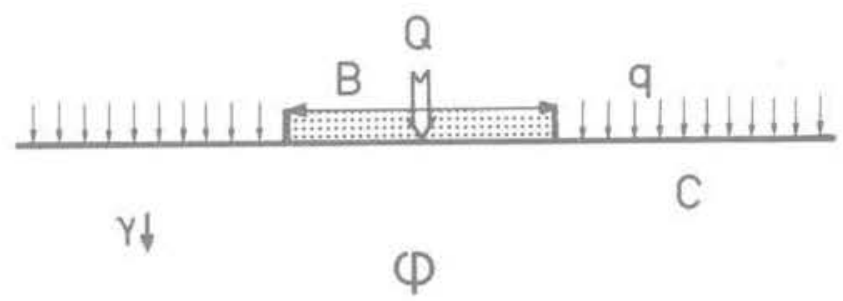

Fig. 3 Semelle filante sur sol illimité homogène

\subsection{Définition de la capacité portante}

II est évidemment nécessaire avant toute discussion sur la validité d'une formule, ou sur les valeurs des coefficients à utiliser, de définir la notion même de capacité portante. On verra d'ailleurs qu'une fois cette définition trouvée et posée les difficultés quant à l'interprétation des solutions et des valeurs proposées par les divers auteurs pour $\mathrm{N}_{\gamma}(\Phi)$ disparaîtront d'elles-mêmes.

On remarque sur la formule (2.3) et en reprenant ce que nous avons dit à ce propos, que la capacité portante de la fondation ne dépend, en ce qui concerne le comportement du sol, que des paramètres $C$ et $\Phi$ qui en caractérisent la " résistance \#. Ceci est révélateur de la démarche logique suivie lorsqu'on traite de capacité portante: on cherche, à partir de la seule connaissance des capacités de résistance du sol au niveau de l'élément (macroscopique de la mécanique des milieux continus), à obtenir une information sur les chargements que pourra supporter la fondation superficielle étudiée ; le chargement considéré est constitué de la surcharge $\mathrm{q}$ et du poids du sol (poids volumique) -éléments stabilisants - et de la charge proprement dite appliquée à la fondation.

Cette démarche a été suivie par tous les auteurs pour les problèmes de capacité portante de fondations. C'est le même type de raisonnement qui est également suivi à propos des problèmes de stabilité de pente depuis Coulomb (1773), et pour les problèmes de poussées et de butées des terres. II s'agit du raisonnement typique du Calcul à la rupture : 
Les capacités de résistance du sol en un point $x$ sont caractérisées par un critère, condition imposée à l'état de contrainte $\sigma(\underline{x})$ en ce point pour pouvoir y être supporté par l'élément de matière et qui est définie par une fonction scalaire $f$ de $\underline{\underline{g}}(\underline{x})\left({ }^{1}\right)$ :

$$
\begin{gathered}
\underline{g}(\underline{x}) \text { supporté par l'élément de sol } \\
\Leftrightarrow f \underline{x} \underline{\underline{x}} \underline{\underline{g}}(\underline{x})) \leqslant 0 ;
\end{gathered}
$$

à partir de cette donnée, on cherche à déterminer pour louvrage étudié la charge maximale qu'il est susceptible de supporter, sans évidemment qu'il soit possible - sauf informations complémentaires sur le comportement - d'affirmer que l'ouvrage supportera effectivement cette charge maximale.

Ceci conduit à la définition de la capacité portante. En effet, en se plaçant dans la géométrie initiale du problème et en la supposant invariable, il est clair que pour que la fondation supporte une charge $Q$, il est nécessaire qu'il existe un champ de contraintes g qui équilibre le chargement constitué par $(Q, \gamma, q)$ en respectant en tout point du milieu (sol et interface) le critère de résistance. On montre alors, sans difficulté, que cette condition nécessaire pour pouvoir être supportée par la fondation, limite les forces $Q$ à un segment de droite $\left[0, \mathrm{Q}^{+}\right]$(figure 4 ) :

On a alors :

$$
\left\{\begin{array}{c}
\begin{array}{l}
\text { F supporté par la fondation (en géométrie } \\
\text { initiale) }
\end{array} \\
\exists \underline{\underline{g}}: \underline{\underline{\sigma}} \text { équilibre }(0, \gamma, q) \text {, et } f \underline{\underline{x}} ; \underline{\underline{\sigma}} \underline{\underline{x}})) \leqslant 0 \forall \underline{\underline{x}} \\
Q \in\left(0, Q_{+}\right)
\end{array}\right.
$$

II est important d'analyser la portée de ce ressultat :

- il est évidemment nécessaire que la géométrie du problème soit donnée et invariable puisque le raisonnement ne se réfère du point de vue du comportement qu'aux "caractéristiques de résistance ", c'est-à-dire au critère de rupture du sol.

(1) Les tenseurs sont soulignés d'un nombre de traits égal à leur ordre. Sans que cela crée d'ambiguités, nous confondrons souvent le champ g et sa valeur locale $g(\underline{x})$
- à partir de ces données le résultat énoncé, qui repose sur la convexité des critères (2.1) et (2.2), est le plus puissant qui puisse être obtenu.

II est alors naturel de définir la capacité portante de la fondation par la valeur moyenne:

$$
\mathrm{q}_{\mathrm{u}}=\mathrm{Q}^{+} / \mathrm{B}
$$

\subsection{Théorème statique ; méthode de superposition}

Il découle immédiatement de cette définition de la capacité portante que la mise en évidence d'un champ de contraintes $\sigma$ statiquement admissible et respectant le critère de résistance du sol en tout point, conduit à une valeur conservative (approche par défaut) de la capacité portante (c'est le théorème statique du calcul à la rupture).

En particulier, en considérant les trois cas de charge représentés sur la figure 5 , et en supposant que l'on dispose des champs de contraintes suivants :

a) $\sigma_{y}$ statiquement admissible et respectant le critère de résistance de l'élément de matière en tout point du milieu pesant, non cohérent et non chargé (figure $5 \mathrm{a}$ ) correspondant à la pression moyenne sous la fondation : $1 / 2 \gamma B N_{,}(\Phi)$

b) $g_{c}$ statiquement admissible et respectant le critère de résistance de l'élément de matière en tout point du milieu cohérent, non pesant et non chargé (figure $5 \mathrm{~b}$ ) correspondant à la pression moyenne sous la fondation : $\mathrm{CN}_{c}(\Phi)$.

c) $\underline{g}_{q}$ statiquement admissible et respectant le critère de résistance de l'élément de matière en tout point du milieu chargé non pesant et non cohérent (figure 5c), correspondant à la pression moyenne sous la fondation: $\mathrm{qN}_{\mathrm{q}}(\Phi)$.

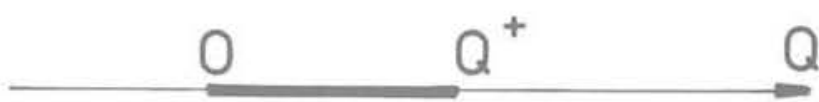

Fig. 4 Forces susceptibles d'être supportées par la fondation dans une géométrie donnée

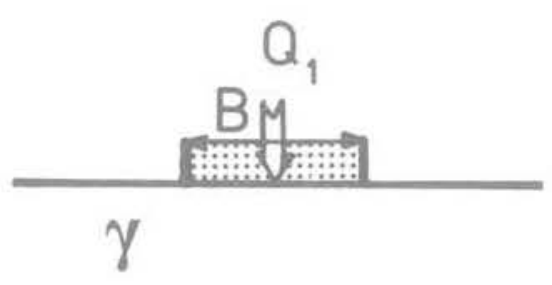

$\varphi$

Sol frottant, pesant non chargé, sans cohésion

(terme de surface)

$$
Q_{1} / B=1 / 2 \gamma B N_{\gamma}(\phi)
$$

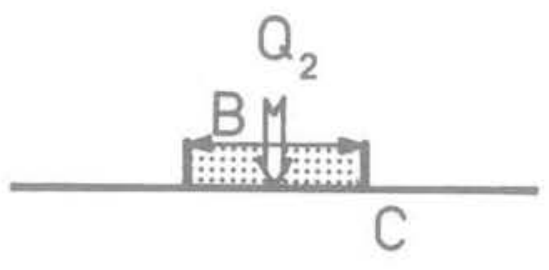

$\varphi$

Sol frottant, avec cohésion, non pesant non chargé (terme de cohésion)

$$
Q_{2} / B=C N_{C}(\phi)
$$

Fig. 5 Méthode de superposition 
On peut vérifier immédiatement, en utilisant, par exemple la représentation de Mohr, que le champ de contraintes (1) :

$$
\underline{\underline{\sigma}}=\underline{\underline{\sigma}}_{\gamma}+\underline{\underline{\sigma}}_{\mathrm{c}}+\underline{\underline{\sigma}}_{\mathrm{q}}
$$

obtenu en additionnant en chaque point du milieu les contraintes produites par $\sigma_{\gamma^{\prime}} \varrho_{c}$, et $g_{q}$, est statiquement admissible et respecte en tout point le critère de résistance pour le sol pesant, cohérent avec surcharge (figure 3 ).

II en résulte, par application du théorème ci-dessus, que pour le problème posé à la figure 3 on $a$ :

(2.7)

$\mathrm{q}_{\mathrm{u}} \geqslant\left(\mathrm{q}_{\mathrm{u}}\right)_{\text {superp. }}=\frac{1}{2} \gamma B \mathrm{~N}_{\gamma}(\Phi)+\mathrm{CN}_{\mathrm{c}}(\Phi)+q N_{\mathrm{q}}(\Phi)$

ce qui démontre le caractère conservatif de la méthode de superposition.

\subsection{Approche cinématique - Solutions complètes} en calcul à la rupture.

On démontre aussi que $\mathrm{q}_{\mathrm{u}}$ peut être approchée par excès commodément par voie cinématique ; ce résultat s'obtient par la dualisation mathématique des équations d'équilibre au moyen du principe des puissances virtuelles et l'utilisation de la fonction d'appui déduite du critère de rupture.

On a ainsi deux approches, qui ressortissent à la terminologie générale d'approches par l'intérieur et par l'extérieur en $\mathrm{Calcul}$ à la rupture ; elles sont analogues aux théorèmes bien connus de l'Analyse limite, mais elles ne font nullement appel à la théorie de la Plasticité. On en trouvera une application détaillée à l'analyse de la stabilité des ouvrages en terre dans (Coussy et Salençon, 1979).

La combinaison de ces deux approches permet d'aboutir ici, par la construction de solutions complètes du point de vue du Calcul à la rupture, à la connaissance de la valeur exacte de $\mathrm{q}_{\mathrm{u}}$.

\subsection{Conséquences}

Une fois posée cette définition précise de la notion de capacité portante qui, comme on l'a vu, correspond très exactement aux idées directrices suivies par les auteurs dans ce domaine, l'interprétation des diverses méthodes proposées pour calculer les valeurs des coefficients figurant dans la formule linéaire (2.3) ne présente aucune difficulté : il suffit d'examiner à quel type d'approche ressortit chacun des raisonnements mis en œuvre. On trouvera dans (Matar, 1978) une analyse détaillée de cette question dont il résulte que $\mathrm{N}_{c}(\Phi)$ et $\mathrm{N}_{0}(\Phi)$ sont donnés par les formules explicites issues de la solution de Prandil complétée par Shield (1954):

$$
\left\{\begin{array}{l}
N_{c}(\Phi)=\operatorname{cotg} \Phi\left[e^{\pi \operatorname{tg} \Phi} \operatorname{tg}^{2}\left(\frac{\pi}{4}+\frac{\Phi}{2}\right)-1\right] \\
N_{q}(\Phi)=e^{\pi \operatorname{tg} \Phi \operatorname{tg}^{2}\left(\frac{\pi}{4}+\frac{\Phi}{2}\right)}
\end{array}\right.
$$

Quant à $N_{\gamma}(\Phi)$ sa valeur est fournie par la solution de Lundgren et Mortensen (1953) complétée par Davis et Booker (1971).

\subsection{Remarques}

On a vu que la seule définition possible pour la capacité portante. $q_{u}$ dans le cadre d'une approche qui ne repose que sur la connaissance des capacités de résistance du sol de fondation, est celle d'une charge extrême au sens du calcul à la rupture: $\mathrm{q}_{\mathrm{u}}$ est la plus grande charge susceptible d'être supportée par la fondation dans les conditions indiquées. II s'agit là évidemment d'une réponse partielle au problème (modélisé) posé, ne serait-ce que parce que rien ne permet d'affirmer que toute charge infé-

(1) Ce résultat repose sur le fait que le domaine des états de contraintes supportables pour le critère de Coulomb est un cône convexe. rieure à $q_{u}$ sera toujours effectivement supportée par la fondation, indépendamment de facteurs tels que trajet de charge, etc. ; de plus, l'importance des déformations avant la rupture, leur rôle éventuel, sont aussi du plus grand intérêt. C'est ce que tentera d'évaluer la comparaison avec les essais sur modèles qui sera évoquée au chapitre 7.

\section{Capacité portante d'une semelle filante sur sol non-homogène. \\ Emploi de la méthode de superposition}

\subsection{Quelques cas de non-homogénéité antérieure- ment étudiés}

La semelle filante sur sol homogène. illimité est évidemment le cas de fondations le plus classique qui ait été étudié. Le petit nombre de paramètres nécessaire à la description du problème du point de vue de la capacité portante réduit le volume des calculs à effectuer dans le cadre de l'application de la méthode de superposition et permet une présentation commode des résultats : tableaux ou courbes pour $N_{y}(\Phi), N_{c}(\Phi), N_{q}(\Phi)$.

Le cas de la semelle filante sur couche de sol homogène d'épaisseur limitée, reposant sur une assise rigide (figure 6) a également été étudié (Mandel et Salençon, 1969 et 1972). Ces auteurs ont appliqué la méthode de superposition en s'appuyant sur le théorème général du § 2.3 et en construisant les solutions complètes des trois problèmes de base, homologues de ceux de la figure 5 .

Avec les notations de la figure 6 l'analyse dimensionnelle montre que les capacités portantes pour les trois problèmes de base s'écrivent:

$$
\begin{aligned}
& \text { a) } \mathrm{q}_{\mathrm{u}}=\frac{1}{2} \gamma \mathrm{BN}\left(\frac{\mathrm{B}}{\mathrm{h}}, \Phi\right) \text {, } \\
& \text { b) } \mathrm{a}_{\mathrm{u}}=\mathrm{CN}_{\mathrm{c}}\left(\frac{\mathrm{B}}{\mathrm{h}}, \Phi\right), \\
& \text { c) } \mathrm{a}_{\mathrm{u}}=\mathrm{qN}_{\mathrm{a}}^{\prime}\left(\frac{\mathrm{B}}{\mathrm{h}}, \Phi\right) \text {; }
\end{aligned}
$$

la capacité portante pour le problème général de la figure 6 est donc évaluée par défaut au moyen de la méthode de superposition sous la forme :

$$
q_{u} \geqslant\left(q_{u}\right)_{\text {superp. }}=\frac{1}{2} \gamma B N_{\gamma}^{\prime}\left(\frac{B}{h}, \Phi\right)+C_{c}^{\prime}\left(\frac{B}{h}, \Phi\right)+q N_{q}\left(\frac{B}{h}, \Phi\right)
$$

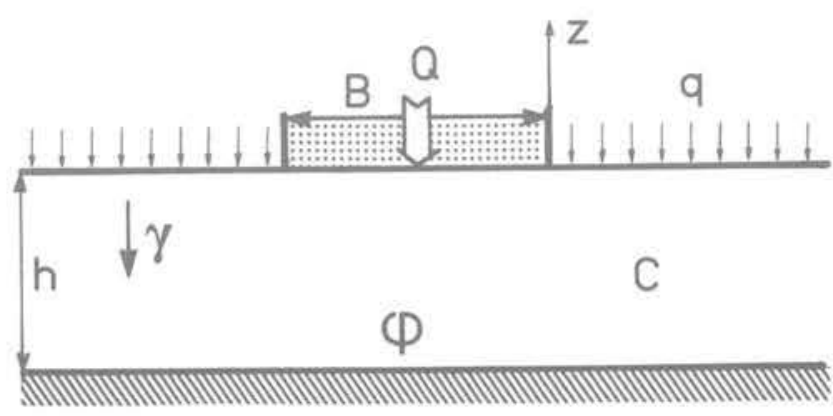

Fig. 6 Semelle filante sur couche de sol homogène d'épaisseur limitée 
Les auteurs ont étudié divers cas de frottement à l'interface entre la couche de sol et son assise rigide, la fondation quant à elle étant supposée parfaitement rugueuse ; dans toute la suite seul le cas où il y a adhérence parfaite entre la couche de sol et l'assise rigide sera considéré.

Les valeurs des coefficients figurant dans (3.2) ont été données dans les références indiquées; elles sont représentées graphiquement dans la suite (figures 10 et 11 ). On a entre $N_{c}^{\prime}\left(\frac{B}{h}, \Phi\right)$ et $N_{q}^{\prime}\left(\frac{B}{h^{\prime}}, \Phi\right)$ la relation : $N_{q}^{\prime}\left(\frac{B}{h}, \Phi\right)=$ $N_{c}\left(\frac{B}{h}, \Phi\right) \operatorname{tg} \Phi+1$

(théorème des états correspondants).

II est à remarquer que, pour chacun des problèmes de base, il existe une valeur critique du rapport $B / h$, soient $(B / h)_{\gamma}$ et $(B / h)_{c}=(B / h)_{q}$, fonctions de $\Phi$ telles que :

$$
\left\{\begin{aligned}
B / h \leqslant(B / h)_{\gamma} & \Leftrightarrow N_{\gamma}^{\prime}\left(\frac{B}{h}, \Phi\right)=N_{\gamma}(\Phi) \\
B / h \leqslant(B / h)_{c} & \Leftrightarrow\left\{\begin{array}{l}
N_{c}^{\prime}\left(\frac{B}{h}, \Phi\right)=N_{c}(\Phi) \\
N_{q}^{\prime}\left(\frac{B}{h}, \Phi\right)=N_{q}(\Phi)
\end{array}\right.
\end{aligned}\right.
$$

c'est-à-dire que l'influence de l'assise rigide ne se fait plus sentir sur la capacité portante de la fondation, lorsque la couche de sol est suffisamment épaisse.

Un autre type de non-homogénéité a été étudié : il s'agit du sol de fondation illimité dont la cohésion croît linéairement avec la profondeur. Ce type de non-homogénéité se rencontre dans la pratique pour les sols à $\Phi<25 \circ$ et plus particulièrement pour les sols à $\Phi=0$. ou voisin de 0 . tels que les sols marins dont Duncan et Buchignani (1973) et le Tirant (1976) ont donné des exemples (figure 7).

L'analyse de ce problème a permis de le traiter par la méthode de superposition et par un calcul global prenant en compte tous les effets de couplage (Salençon, Florentin et Gabriel, 1976). II n'est pas nécessaire d'en détailler ici les résultats : comme les précédents ce sont des cas particuliers des résultats de la présente étude.

Enfin, récemment, Matar et Salençon (1977) ont publié des résultats sur la couche de sol purement cohérent et de cohésion linéairement variable avec la profondeur.

Les travaux mentionnés ci-dessus sont ceux qui se rattachent directement, par les méthodes utilisées, à l'étude présentée ici. D'autres auteurs se sont intéressés à ces problèmes et les ont abordés par d'autres voies; nous renvoyons le lecteur à Obin (1972) et à Giroud, Tran-VoNhiem et Obin (1973) pour une bibliographie détaillée: l'interprétation des solutions devra se faire par référence aux théorèmes des $\$ 2.3$ et 2.4 .

\subsection{Le problème étudié (problème P)}

On se propose maintenant d'étudier la capacité portante d'une semelle filante de largeur B, chargée axialement, reposant sur une couche de sol d'épaisseur limitée $h$, de poids volumique $\gamma$, d'angle de frottement interne $\Phi$ constant et dont la cohésion croît linéairement avec la profondeur selon la loi :

$$
\mathrm{C}(\mathrm{z})=\mathrm{C}_{\mathrm{o}}-\mathrm{gz}
$$

$C_{0}$ est la cohésion en surface et $\mathrm{g}=-\mathrm{dC} / \mathrm{dz}(>0)$ est le gradient de cohésion avec la profondeur, $\mathrm{C}_{0}$ et $\mathrm{g}$ sont constants.

La couche de sol repose sur une assise rigide avec une surcharge latérale q (figure 8).

Nous supposons dans toute la suite qu'il s'agit d'une semelle filante et d'une assise rigide rugueuses. Par
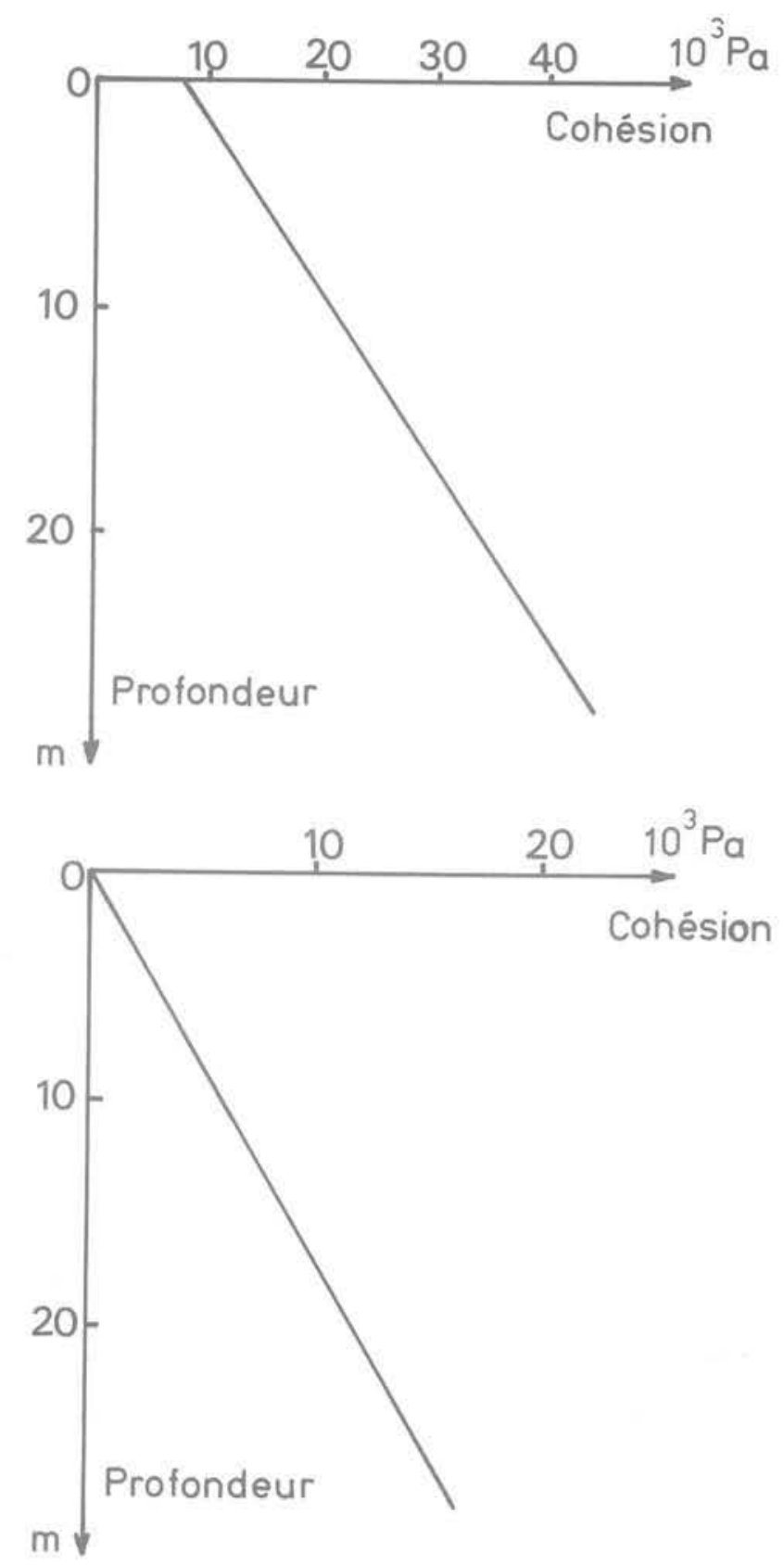

Fig. 7 Exemples de résistance au cisaillement des sols marins

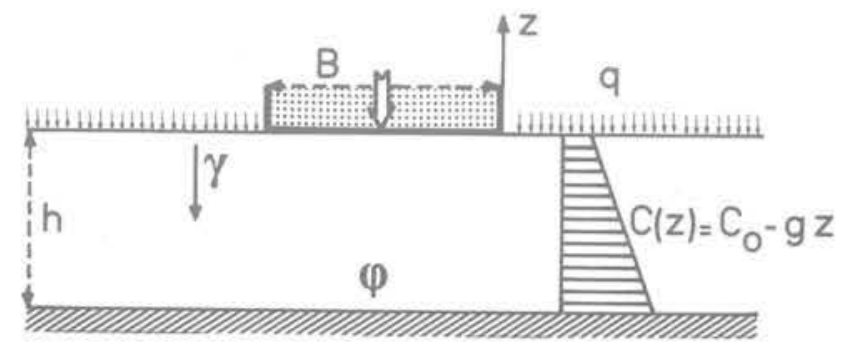

Fig. 8 Problème $P$; sol pesant, cohésion variable 
semelle (resp. assise) rugueuse, on entend que l'adhérence est totale entre la semelle et le sol, c'est-à-dire que toute rupture se produit dans le sol et non pas dans l'interface sol-semelle (resp. sol-assise).

Le problème étant ainsi posée ${ }^{(1)}$ la capacité portante de la semelle est a priori une fonction des paramètres $\mathrm{C}_{0}, \mathrm{~g}, \mathrm{~B}$, h, $\gamma, \Phi, \mathbf{q}$ :

(3.5) $q_{u}=f\left(C_{o^{\prime}} g, B, h, \gamma, \Phi, q\right)$

3.3 Sol frottant; utilisation de la méthode de superposition

On va voir, par une analyse mécanique du problème, que dans le cas d'un sol frottant $(\Phi \neq 0)$ une évaluation par défaut de la capacité portante peut être obtenue à partir de la formule (3.2), c'est-à-dire par une application de la méthode de superposition, sans qu’il soit nécessaire de procéder à des calculs nouveaux.

On démontre en effet le théorème suivant:

" équivalence $\mathrm{P}-\mathrm{P}^{1}$ "

Pour un sol à $\Phi \neq 0$, la capacité portante $q_{u}$ pour le problème $P$ se déduit par la formule:

$q_{u}=q_{u}^{1}+q$

de la capacité portante $q_{u}^{t}$ correspondant au problème $P^{l}$ géométriquement identique au précédent défini comme suit: sol homogène, de même angle de frottement $\Phi$, de poids volumique $\gamma^{1}=\gamma+g \operatorname{cotg} \Phi$, et de cohésion $C^{1}=C_{0}+q \operatorname{tg} \Phi$.

Cette propriété est analogue à celles énoncées par Salencon, Barbier et Beaubat (1973) et Salençon, Florentin et Gabriel (1976).

La figure 9 donne les détails de la correspondance entre les deux problèmes.

II en résulte alors, de façon évidente par (3.2), l'évaluation par défaut de la capacité portante fournie par une application de la méthode de superposition au problème $\mathrm{P}^{\prime}$;

$$
\mathrm{q}_{u} \geqslant\left(\mathrm{q}_{u}^{1}\right)_{\text {superp }}+\mathrm{q}=\frac{1}{2}(\gamma+\mathrm{g} \operatorname{cotg} \Phi) \mathrm{BN}_{\gamma}^{\prime}\left(\frac{\mathrm{B}}{\mathrm{h}}, \Phi\right)
$$

$+\left(C_{0}+q \operatorname{tg} \Phi\right) N_{c}\left(\frac{B}{h}, \Phi\right)+q=\left(q_{u}\right)_{\text {superp. }}$

(1) II est clair que ce problème contient, comme cas particuliers, tous ceux qui ont été évoqués auparavant

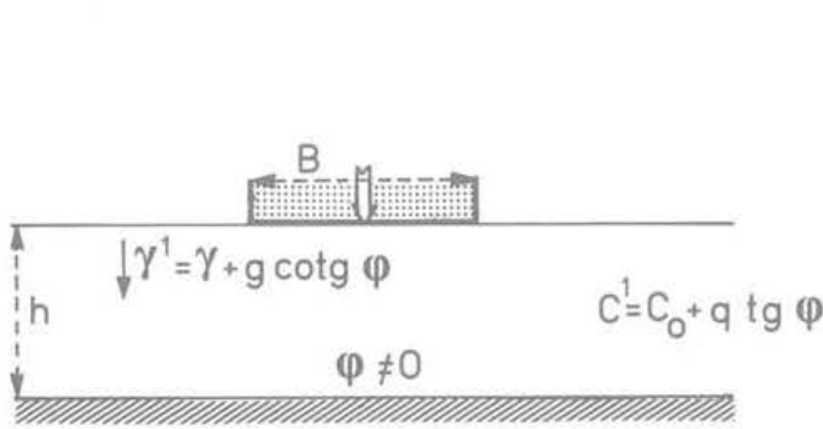

Problème $P^{1}$

Sol pesant, poids spécifique modifié, cohésion constante modifiée
On voit ainsi qu'une simple analyse mécanique du problème posé permet, sans avoir à effectuer des calculs nouveaux, d'obtenir une évaluation par défaut non triviale de la capacité portante, prenant en compte l'effet de gradient de cohésion dans le cas d'une couche de sol frottant.

\subsection{Sol purement cohérent}

II est évident, par la présence du facteur cotg $\Phi$ dans les formules de correspondance $P-P^{1}$ indiquées plus haut, que la méthode ci-dessus ne peut s'appliquer directement dans le cas d'un sol purement cohérent $(\Phi=0)$. II est nécessaire de procéder alors à une analyse particulière.

On démontre les deux propriétés suivantes (cf. Matar et Salençon, 1977):

a) $\mathrm{Si}_{0}=0$, la capacité portante de la semelle est :

$$
\mathrm{q}_{\mathrm{u}}=\frac{1}{4} \mathrm{gB}+\mathrm{q}, \quad \forall \mathrm{B} / \mathrm{h} \text {; }
$$

b) dans le cas général pour le sol purement cohérent $(\Phi=0)$, la superposition de la capacité portante correspondant à une cohésion constante égale à $\mathrm{C}_{\mathrm{O}}$ et à la surcharge $q^{(1)}$, et de celle due au gradient de cohésion va dans le sens de la sécurité :

$$
\mathrm{q}_{\mathrm{u}} \geqslant\left(\mathrm{q}_{\mathrm{u}}\right)_{\text {superp. }}=\mathrm{C}_{\mathrm{o}} \mathrm{N}_{\mathrm{c}}^{\prime}\left(\frac{\mathrm{B}}{\mathrm{h}}, \mathrm{o}\right)+\frac{1}{4} \mathrm{~g} B+\mathrm{q}
$$

La formule (3.9) fournit ainsi une évaluation par défaut de la capacité portante de la semelle pour $\Phi=0$, prenant en compte l'effet du gradient de cohésion ; il s'agit d'une formule de superposition. De plus cette formule est bien l'homologue de la formule (3.7) en ce sens que la continuité entre ces deux formules quand $\Phi \rightarrow 0$ correspond à la propriété :

$\mathrm{N}_{\gamma}^{\prime}\left(\frac{\mathrm{B}}{\mathrm{h}}, \Phi\right) \sim \Phi / 2$ quand $\Phi \rightarrow 0, \forall \mathrm{B} / \mathrm{h}$.

\subsection{Commentaires}

Le théorème "d'équivalence $P-P^{1}$ 》 énoncé au $\$ 3.3$ met en évidence pour le matériau frottant :

d'une part une équivalence entre les effets de la cohésion $\mathrm{C}_{0}$ et de la surcharge $\mathrm{q}$ : c'est le théorème des "états correspondants » :

d'autre part une équivalence entre les effets du gradient vertical de cohésion et du poids volumique $\gamma$ : du point de vue de la capacité portante, le gradient vertical de cohésion g est équivalent à un poids volumique additionnel égal à g cotg $\Phi$; nous aurons l'occasion d'utiliser cette équivalence, pour ainsi dire "en sens inverse " au chapitre suivant.

(1) Pour $\Phi=0$, la surcharge $q$ a un effet purement additif

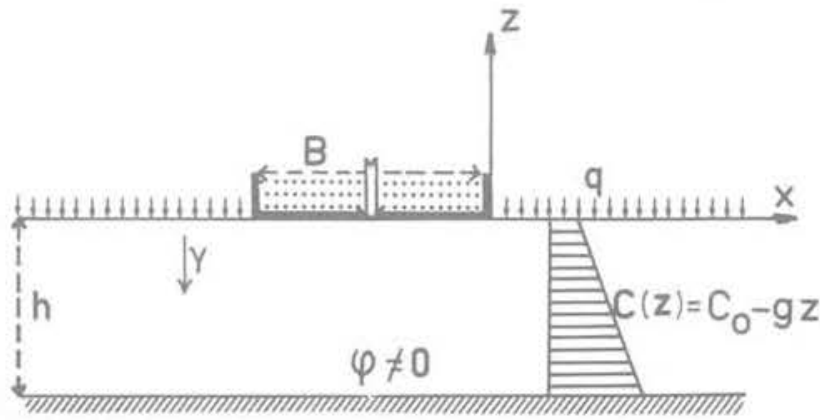

Problème $P$

Sol pesant, cohésion variable

Fig. 9 Problèmes $P^{1}$ et $P$;

correspondance entre les champs de contraintes : $\underline{\underline{\sigma}}^{1}=\underline{\underline{\sigma}}-(g z \operatorname{cotg} \phi+q) \underline{\underline{1}}$;

correspondance entre les capacités portantes: $q_{u}^{1}=q_{u}=q$ 

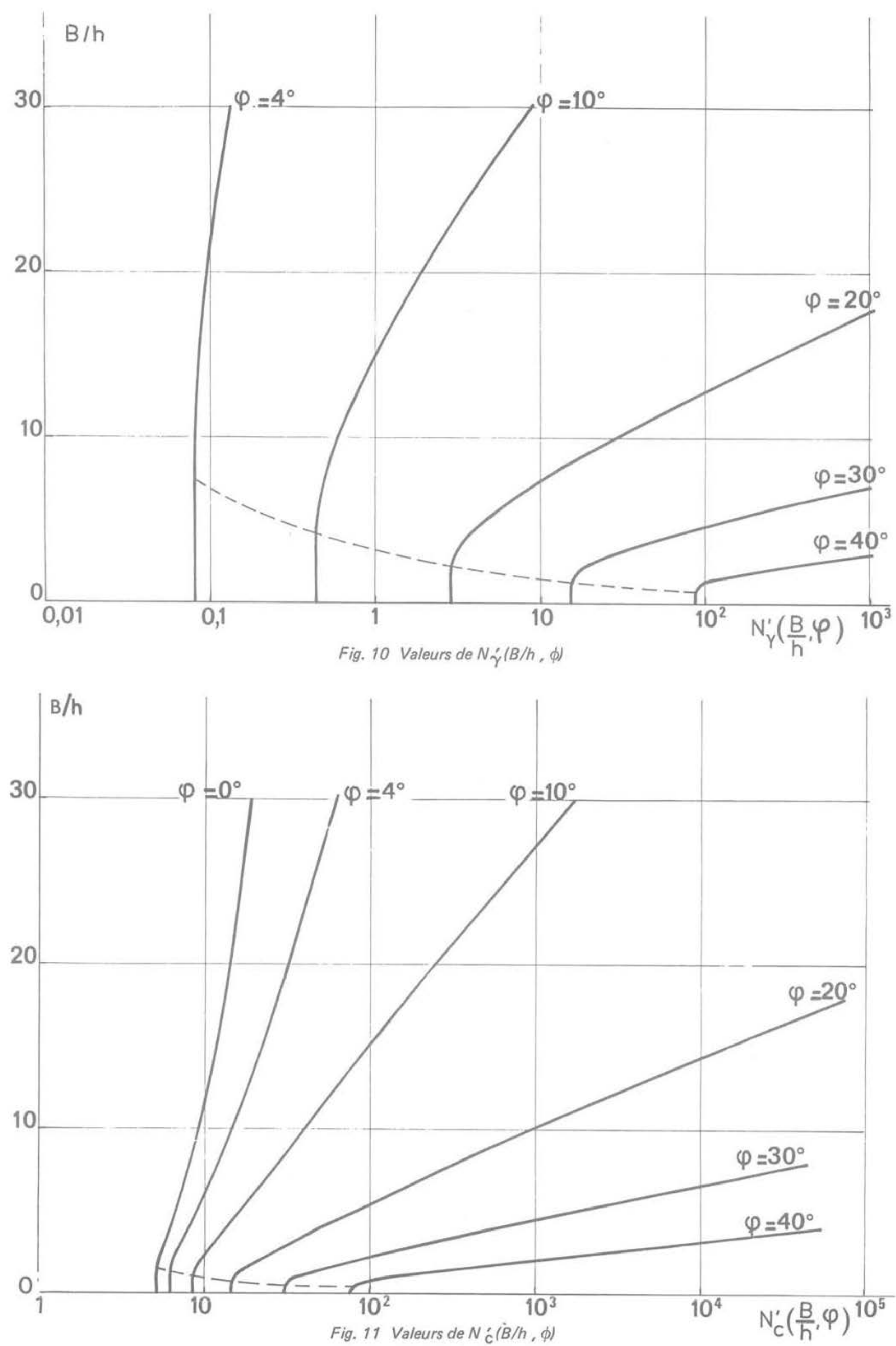
Ici ces deux équivalences ont conduit à des regroupements de termes à partir desquels il a été possible d'appliquer la formule (3.2). Les figures 10 et 11 représentent les

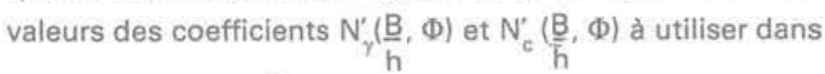
(3.7) ainsi que $N_{c}^{\prime}\left(\frac{B}{h}, 0\right)$ pour (3.9).

Comme on l'a dit, l'évaluation par défaut (3.7) résulte de l'application de la méthode de superposition au problème $\mathrm{P}^{1}$. On pourrait être tenté, suivant le théorème général du § 2.3. d'appliquer cette méthode directement au problème $\mathrm{P}$; comme cela est expliqué dans (Matar, 1978) une telle application nécessiterait d'effectuer des calculs nouveaux qui seraient en fait identiques à ceux qui seront mis en cuvre au chapitre suivant pour le calcul global de la capacité portante pour ce même problème $P$ : cette application de la méthode de superposition au problème $\mathrm{P}$ serait donc sans intérêt réel.

\section{Capacité portante d'une semelle filante sur sol non-homogène. Calcul global}

4.1 Position du problème : "équivalence $\mathrm{P}-\mathrm{P}^{2}$ " II est commode pour l'analyse qui va être faite dans la suite, de reprendre "en sens inverse " le raisonnement développé à propos de l' "équivalence $P-P^{1}$ " au $\$ 3.3$. On démontre ainsi le théorème suivant :

"équivalence $\mathrm{P}-\mathrm{P}^{2}$ 》

La détermination de la capacité portante $q_{u}$ pour le problème $P$ se ramène, par la formule

$$
\mathrm{q}_{\mathrm{u}}=\mathrm{q}_{\mathrm{u}}^{2}+\mathrm{q}
$$

à la détermination de la capacité portante $q_{u}^{2}$ pour le problème $P^{2}$, géométriquement identique au précédent et défini comme suit : sol non pesant, d'angle de frottement $\Phi$, de cohésion variable

$C^{2}(z)=\left(C_{0}+q \operatorname{tg} \Phi\right)-(g+\gamma \operatorname{tg} \Phi) z$, sans surcharge.

La figure 12 détaille la correspondance entre ces deux problèmes tandis que le tableau 1 récapitule les positions de $P, P^{1}$ et $P^{2}$

On remarque que, dans cet énoncé, ne figure aucune restriction sur $\Phi$; en effet la démonstration demeure valable pour $\Phi=0$. On peut donc ainsi regrouper sous le même formalisme les cas du sol purement cohérent et du sol frottant $\left({ }^{1}\right)$.

On déduit immédiatement de ce théorème d'équivalence que la capacité portante $\mathrm{q}_{4}$ ne dépend des paramètres définissant le problème $P$ indiqués à la formule $(3.5)$ que par l'intermédiaire des groupements définissant le problème $P^{2}$. Plus précisément, compte tenu de $(4.1)$ et avec la notation de (3.5) on a :

$\mathrm{q}_{\mathrm{u}}=\mathrm{q}+\mathrm{f}\left(\mathrm{C}_{\mathrm{o}}+\mathrm{q} \operatorname{tg} \Phi, \mathrm{g}+\gamma \operatorname{tg} \Phi, \mathrm{B}, \mathrm{h}, 0, \Phi, 0\right)$

Par l'analyse dimensionnelle, on déduit alors que la capacité portante $\mathrm{q}_{4}$ pour le problème $P$ se met nécessairement sous les formes suivantes:

$$
\begin{aligned}
& \left\{\begin{array}{l}
\mathrm{q}_{\mathrm{u}}=\mathrm{q}+\left(\mathrm{C}_{0}+\mathrm{q} \operatorname{tg} \Phi\right) \mathrm{F}_{\mathrm{C}}\left(\frac{\mathrm{g}+\gamma \operatorname{tg} \Phi}{\mathrm{C}_{0}+q \operatorname{tg} \Phi} \mathrm{B}, \frac{\mathrm{B}}{\mathrm{h}}, \Phi\right) \\
\text { si }\left(\mathrm{C}_{0}+\mathrm{q} \operatorname{tg} \Phi\right) \neq 0
\end{array}\right. \\
& \left\{\begin{array}{l}
\mathrm{q}_{\mathrm{u}}=\mathrm{q}+(\mathrm{g}+\gamma \operatorname{tg} \Phi) \mathrm{B}, \mathrm{K}\left(\frac{\mathrm{B}}{\mathrm{h}}, \Phi\right) \\
\text { si }\left(\mathrm{C}_{0}+\mathrm{q} \operatorname{tg} \Phi\right)=0
\end{array}\right.
\end{aligned}
$$$$
\text { (4.3) }
$$

(1) Ceci prouve en fait que c'est le facteur $N_{g}^{\prime}=N^{\prime}, \operatorname{cotg} \Phi$ qui est significatif et non $\mathrm{N}_{\text {y }}^{*}$ lui-même (de la même maniêre que $\mathrm{N}_{\mathrm{c}}=\left(\mathrm{N}_{\mathrm{q}}-1\right)$ cotg $\Phi$ et non $\mathrm{N}_{\mathrm{q}}-1$ ). Ceci apparaitra dans les abaques pour le calcul pratique donnés au chapitre 5 . où $F_{c}$ et $\mathrm{K}$ sont des fonctions scalaires des arguments indiqués.

L'intérêt de ces formules est évident: la réduction du nombre de paramètres dont dépend effectivement $q_{u^{\prime}}$ d'abord par l'analyse mécanique, puis par l'analyse dimensionnelle, diminue évidemment de manière considérable le volume des calculs à effectuer pour la détermination complète de $q_{u}$. On comprend également que la présentation des résultats en sera grandement facilitée.

\subsection{Détermination de la fonction $\mathrm{K}(\mathrm{B} / \mathrm{h}, \Phi)$}

La formule (4.3) correspondant au cas où $\left(\mathrm{C}_{0}+\mathrm{qtg} \Phi\right)=0$, la détermination de la fonction $\mathrm{K}(\mathrm{B} / \mathrm{h}, \Phi)$ se ramène au calcul de $q_{u}^{2}$ pour le problème $P^{2}$ dans le cas où la cohésion $C^{2}(z)$ est nulle en surface: $C^{2}(0)=0$.

- Supposons d'abord $\Phi \neq 0 ; q_{u}^{2}$ est alors égale à $q_{u}^{1}$ pour le problème $\mathrm{P}^{\mathrm{t}}$ homologue (tableau 1 ), c'est-à-dire où la cohésion en surface est nulle. Ce problème n'est autre que l'un des trois problèmes de base évoqués au § 3.1 pour la semelle filante sur couche de sol homogène : c'est le problème a) et l'on $a$, en se référant à (3.1):

$q_{u}=\frac{1}{2}(\gamma+g \operatorname{cotg} \Phi) B N_{\gamma}(B / h, \Phi)$

On en déduit :

$$
\left\{\begin{array}{l}
\mathrm{K}(\mathrm{B} / \mathrm{h}, \Phi)=\frac{1}{2} \mathrm{~N}_{\gamma}(\mathrm{B} / \mathrm{h}, \Phi) \operatorname{cotg} \Phi \\
\text { si } \Phi \neq 0
\end{array}\right.
$$

- Pour $\Phi=0$, le problème $P^{2}$ à étudier est celui de la semelle filante sur couche de sol purement cohérent d'épaisseur limitée, de cohésion nulle en surface et croissant linéairement avec la profondeur selon le gradient $\mathrm{g}^{2}=\mathrm{g}+\gamma \operatorname{tg} \Phi$. C'est le problème évoqué au $\$ 3.4$ et la capacité portante y est donnée par (3.8), d'où iri:

$\mathrm{q}_{\mathrm{u}}=\frac{1}{4}(\mathrm{~g}+\gamma \operatorname{tg} \Phi) \mathrm{B}=\frac{1}{4} \mathrm{gB}$

On en déduit :

$$
K\left(\frac{B}{h}, 0\right)=\frac{1}{4}
$$

La fonction $K(\underline{B}, \Phi)$ est ainsi déterminée pour $\Phi \geqslant 0$ sans qu'il soit nécessaire de procéder à aucun calcul nouveau.

\subsection{Détermination de la fonction}

$\mathrm{F}_{\mathrm{c}}\left(\frac{\mathrm{g}+\gamma \operatorname{tg} \Phi}{\mathrm{C}_{\mathrm{o}}+\mathrm{q} \operatorname{tg} \Phi} \mathrm{B}, \frac{\mathrm{B}}{\mathrm{h}}, \Phi\right)$

La détermination de la fonction $F$ s'effectue en construisant, pour $\left(C_{0}+q \operatorname{tg} \Phi\right) \neq 0$, une solution complète pour le problème $\mathrm{P}^{2}$. On s'appuie pour cela sur la théorie des équilibres limites plans pour les sols non-homogènes (cf. par ex. Olszak, Rychlewski et Urbanowski (1962) ou Salençon (1974) pour les équations de ce problème).

Nous nous restreindrons ici à donner des indications sommaires sur la démarche suivie et la forme des réseaux de lignes caractéristiques obtenus. Tous les détails sont disponibles dans la référence (Matar, 1978) déjà citée, en particulier en ce qui concerne les champs de vitesse et les prolongements des champs de contraintes.

La construction de la solution pour une couche de so d'épaisseur limitée s'effectue à partir de la solution du cas du sol semi-infini $(\mathrm{h}=\infty)$ d'une manière analogue à celle décrite dans Mandel et Salençon $(1969,1972)$ et Matar et Salençon (197I). Elle a été faite dans I'hypothèse $(\mathrm{g}+\gamma \operatorname{tg} \Phi) \geqslant 0$. 


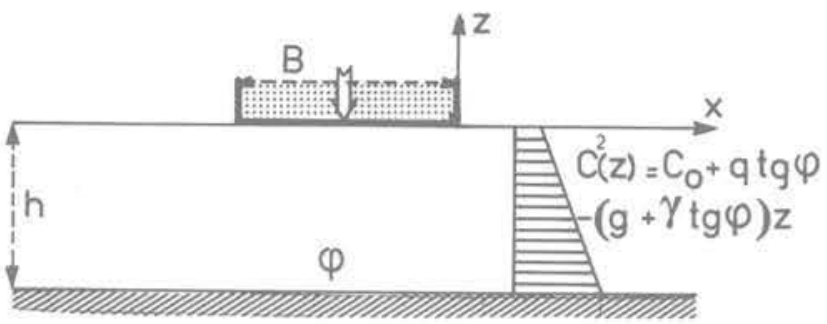

Problème $P^{2}$

Sol non pesant, cohésion variable modifiée

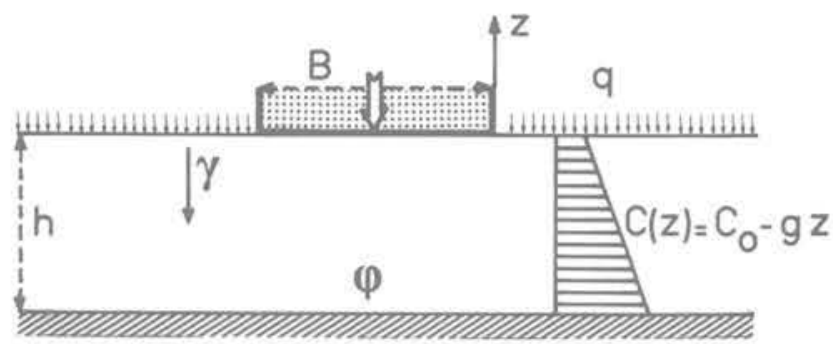

Problème P

Sol pesant, cohésion variable

Fig. 12 Problèmes $P^{2}$ et $P$;

correspondance entre les champs de contrainte: $\underline{\underline{\sigma}}^{2}=\underline{\underline{\sigma}}+(\gamma z-q) \underline{\underline{1}}$;

correspondance entre les capacités portantes : $q_{u}^{2}=q_{u}-q$

\begin{tabular}{|c|c|c|c|}
\hline Problème & $\Phi \geqslant 0$ & $\Phi>0$ & $\Phi \geqslant 0$ \\
\hline $\begin{array}{l}\text { Numéro de la } \\
\text { figure }\end{array}$ & 9 et 12 & 9 & 12 \\
\hline $\begin{array}{l}\text { Poids } \\
\text { volumique }\end{array}$ & $\gamma$ & $\gamma^{1}=\gamma+\mathrm{g} \operatorname{cotg} \Phi$ & 0 \\
\hline $\begin{array}{l}\text { Gradient de } \\
\text { cohésion }\end{array}$ & g & 0 & $\mathrm{~g}^{2}=\mathrm{g}+\gamma \operatorname{tg} \Phi$ \\
\hline $\begin{array}{l}\text { Cohésion en } \\
\text { surface }\end{array}$ & $\mathrm{C}_{0}$ & $C_{0}+q \operatorname{tg} \Phi$ & $C_{0}+q \operatorname{tg} \Phi$ \\
\hline $\begin{array}{l}\text { Surcharge } \\
\text { latérale }\end{array}$ & $q$ & 0 & 0 \\
\hline $\begin{array}{l}\text { Capacité } \\
\text { portante }\end{array}$ & $q_{u}$ & $q_{u}^{1}=q_{u}-q$ & $\mathrm{q}_{\mathrm{u}}^{2}=\mathrm{q}_{\mathrm{u}}-\mathrm{q}$ \\
\hline $\begin{array}{l}\text { Champ de } \\
\text { contrainte }\end{array}$ & $\underline{\sigma}$ & $\underline{\underline{\sigma}}^{1}=\underline{\underline{\sigma}}-(\mathrm{gz} \operatorname{cotg} \Phi+\mathrm{q}) \underline{\underline{1}}$ & $\underline{\underline{\sigma^{2}}}=\underline{\underline{\sigma}}+(\gamma z-\mathrm{q}) \underline{\underline{1}}$ \\
\hline
\end{tabular}

Tableau 1 Position des problèmes $P, P^{1}$ et $P^{2}$

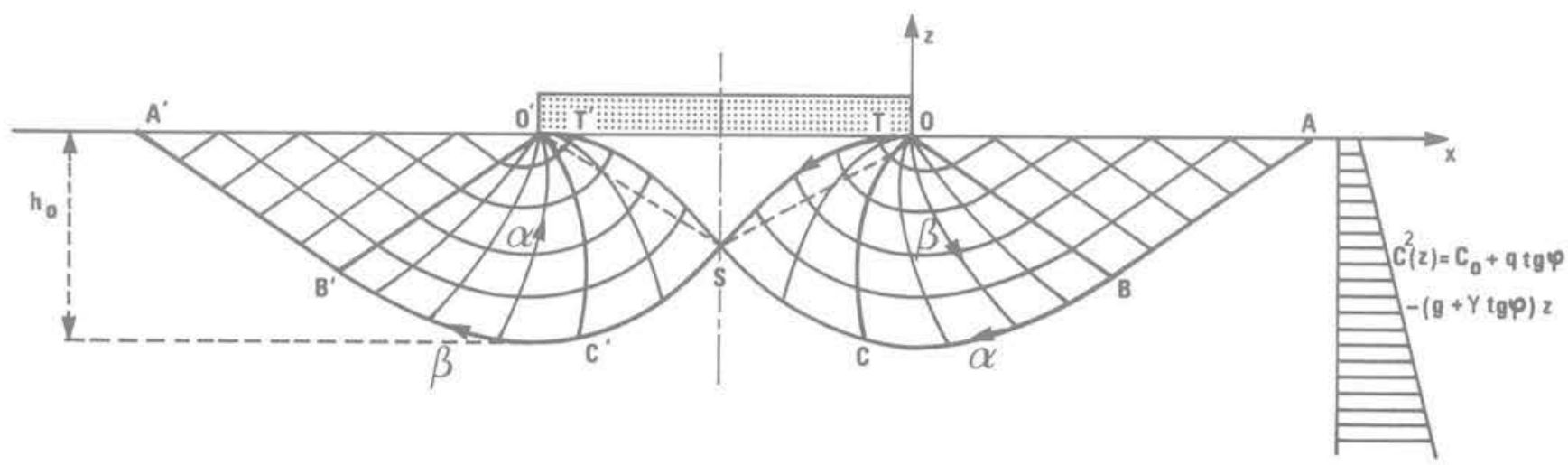

Fig. 13 Fondations sur sol d'épaisseur illimitée ; réseau de caractéristiques 
- Sol illimité $(\mathrm{h}=0)$

La figure 13 représente un réseau de caractéristiques tel que construit par Berthet, Hayot et Salençon (1972) pour un sol purement cohérent $(\Phi=0)$, et Salençon, Barbier et Baubat (1973) pour un sol frottant $(\Phi \neq 0)$.

\section{Ce réseau se compose :}

$1^{\circ}$ d'un champ de Rankine en milieu non-homogène: $O A B$,

$2^{\circ}$ d'un éventail de Prandtl en milieu non-homogène, centré en 0 , d'ouverture $3 \pi / 4+\Phi / 2$ : OBC.

$3^{\circ}$ la construction du réseau est ensuite poursuivie par les caractéristiques $(a)$ s'appuyant sur $O C$ et les caractéristiques $(\beta)$ tangentes à $\mathrm{O}^{\prime} \mathrm{O}$ en conséquence de la rugosité de la fondation. Le réseau des caractéristiques ainsi construit, est évidemment symétrique par rapport à l'axe de la fondation et est alors limité par les caractéristiques SCBA $(\alpha)$ et ST ( $\beta)$ (et symétriques) qui se coupent sur l'axe de symétrie en un point $\mathrm{S}$, de façon à ce que la direction principale des contraintes correspondant à la compression maximale soit verticale. Cette condition détermine la longueur de $O A$ et les autres dimensions du réseau. inconnues a priori.

Le réseau ainsi construit, (figure 13), s'enfonce dans le sol jusqu'à la profondeur $h_{0}$, proportionnelle a $B$ et fonction de:

$\frac{g+\gamma \operatorname{tg} \Phi}{C_{0}+q \operatorname{tg} \Phi} B \quad$ et $\Phi$.

La figure 14 représente $h_{d} / B$ en fonction de ces arguments.

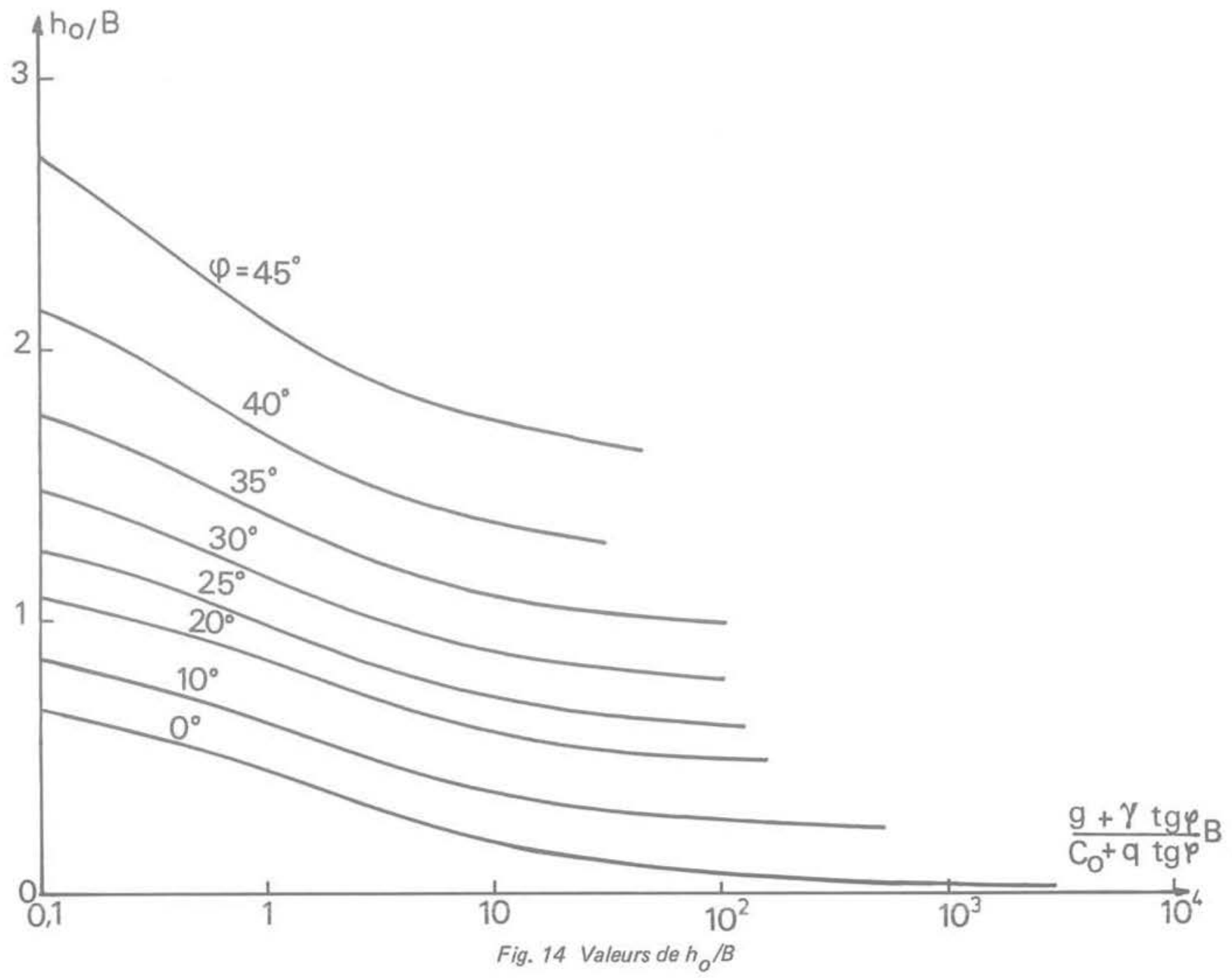

Pour $(\mathrm{g}+\gamma \operatorname{tg} \Phi)=0$, on a $\mathrm{B} / \mathrm{h}_{\mathrm{o}}=(\mathrm{B} / \mathrm{h})_{\mathrm{c}}$ correspondant à un sol homogène cohérent non pesant ; pour $\left(C_{0}+q \operatorname{tg} \Phi\right)=0$ on a $B / h_{0}=(B / h)_{y}$ cf. formule (3.3). Comme on le voit $h_{o} / B$ est une fonction décroissante de $(g+\gamma \operatorname{tg} \Phi) B /\left(C_{0}+q \operatorname{tg} \Phi\right)$ à $\Phi$ donné. Cela correspond au fait que, à cohésion en surface $C_{0}$ et surcharge q données, le sol est perturbé d'autant moins profondément qu'il devient plus vite plus résistant, c'est-à-dire que le gradient $\mathrm{g}^{2}=(\mathrm{g}+\gamma \operatorname{tg} \Phi)$ est plus grand.

La solution ainsi construite permet de déterminer :

$F_{c}\left(\frac{g+\gamma \operatorname{tg} \Phi}{C_{0}+q \operatorname{tg} \Phi}\right.$ B, O, $\left.\Phi\right)$

- Couche de sol d'épaisseur $h \geqslant h$ 。

Pour une couche de sol d'épaisseur limitée $h \geqslant h_{0}$, la construction du réseau de caractéristiques de la figure 13 peut être reprise sans changement (figure 15).

En conclusion :

a $B, \frac{g+\gamma \operatorname{tg} \Phi}{C_{0}+q \operatorname{tg} \Phi} B$ et $\Phi$ donnés, si $h \geqslant h_{0}$

l'assise rigide n'a aucune influence sur la capacité portante de la semelle filante.

Autrement dit :

$$
\left\{\begin{array}{l}
F_{c}\left(\frac{g+\gamma \operatorname{tg} \Phi}{C_{o}+q \operatorname{tg} \Phi} B, \frac{B}{h}, \Phi\right)=F_{c}\left(\frac{g+\gamma \operatorname{tg} \Phi}{C_{o}+q \operatorname{tg} \Phi}, B, O, \Phi\right) \\
\text { si } O \leqslant \frac{B}{h} \leqslant \frac{B}{h}
\end{array}\right.
$$




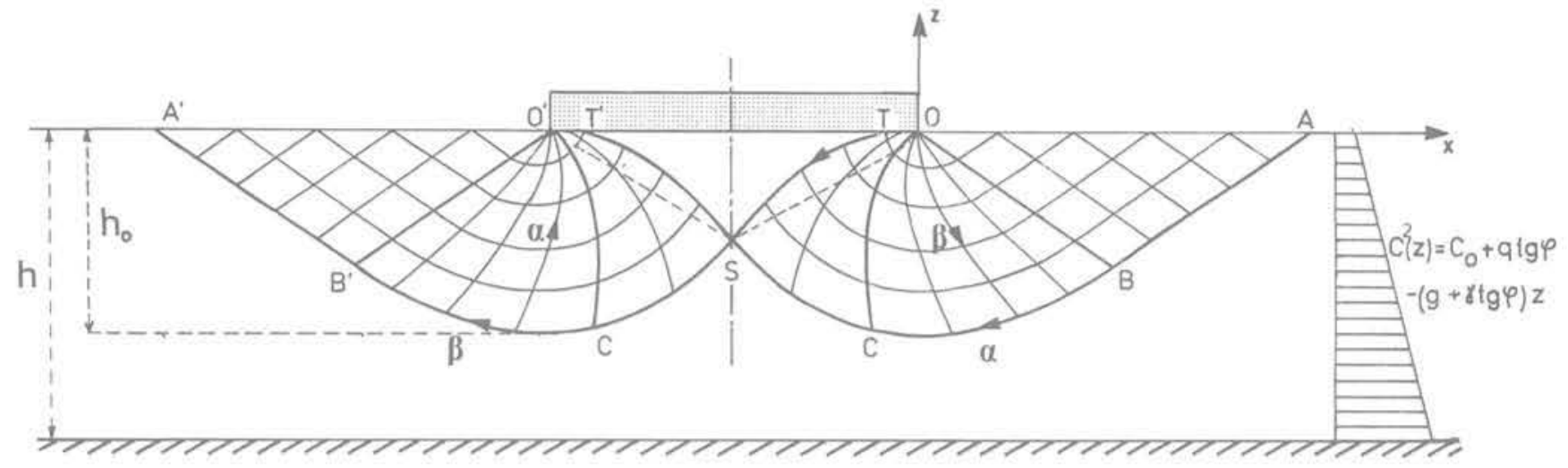

Fig. 15 Fondation sur une couche d'épaisseur $h \geqslant h_{0}$

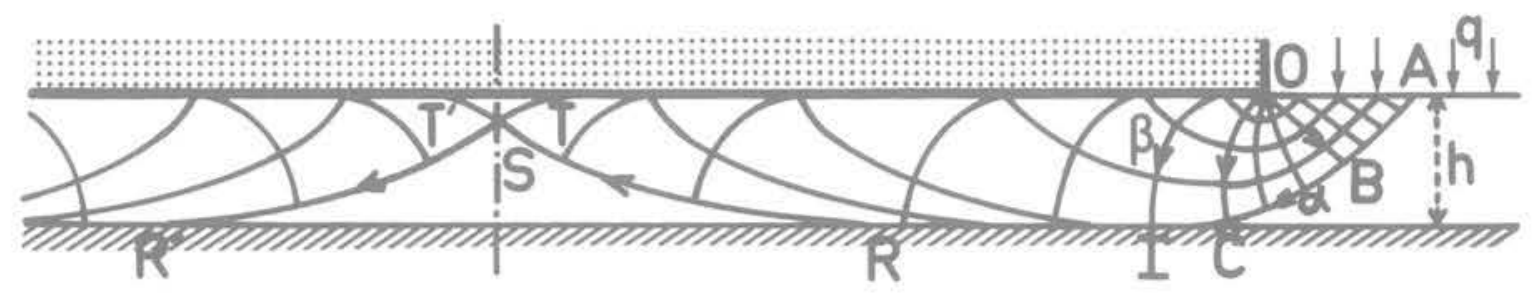

Fig. 16 Fondation sur couche $h \leqslant h_{0}$, réseau de caractéristiques

- Couche de sol d'épaisseur $h \leqslant h$ 。

Le réseau de caractéristiques de la figure 15 n'est évidemment pas utilisable pour une couche de sol d'épaisseur h inférieure à $h$. Dans ce cas, la construction de la solution, symétrique par rapport à l'axe de la fondation, s'effectue comme cela est présenté sur la figure 16.

$1 \mathrm{OAB}$ est un champ de Rankine en milieu non homogène :

2 OBC : un éventail de Prandtl en milieu non homogène centré en $O$ et d'ouverture $(3 \pi / 4+\Phi / 2)$;

3 La construction du réseau de caractéristiques au-delà de ces champs se poursuit à partir de $\mathrm{OC}$ et $\mathrm{Cl}$ en s'appuyant sur les conditions aux limites.

Le réseau est limité par les caractéristiques SR et ST qui se coupent sur l'axe de la fondation comme dans le cas d'un sol illimité et est complété par symétrie.

II reste, dans ces diverses solutions, à calculer la capacité portante $q_{u}^{2}$. Celle-ci est l'intégrale de la contrainte verticale (correspondant à la solution construite) sous la fondation divisée par la largeur B de celle-ci :

(4.7) $q_{u}^{2}=\frac{1}{B} \int_{-B}^{0} \sigma_{z z}(x, 0) d x$

Pour calculer $q_{u}^{2}$, il suffit alors d'intégrer les contraintes connues sur STO et d'en prendre la composante verticale qui représente la valeur $\mathrm{q}_{\mathrm{u}}^{2}, \mathrm{~B} / 2$.

Sur les figures 17,18 et 19 nous représentons par des courbes (iso-B/h) les valeurs de:

(4.8) $F_{c}\left(\frac{g+\gamma \operatorname{tg} \Phi}{C_{0}+q \operatorname{tg} \Phi} B, \frac{B}{h}, \Phi\right)=\frac{q_{u}-q}{C_{0}+q \operatorname{tg} \Phi}=\frac{q_{u}^{2}}{C_{0}+q \operatorname{tg} \Phi}$
4.4 Comparaison avec la méthode de superposition Les formules (4.2) et (4.3) jointes à la connaissance des fonctions $K\left(\frac{B}{h}, \Phi\right)$ et $F_{c}\left(\frac{g+\gamma \operatorname{tg} \Phi}{C_{0}+q \operatorname{tg} \Phi} B, \frac{B}{h}, \Phi\right)$ fournie par (4.4) et (4.5) d'une part et par les coubes telles que celles des figures 17 à 19 pour les diverses fournie par (4.4) et (4.5) d'une part et par les coubes telles que celles des figures 17 à 19 pour les diverses valeurs de $\Phi$, permettent de calculer la valeur exacte de la capacité portante pour le problème $P$, c'est à dire $q_{4}$.

Cette valeur exacte, obtenue par un calcul global, tient compte des effets de couplage possibles entre les divers paramètres définissant le problème. Les analyses mécanique et dimensionnelle faites au § 4.1, par les regroupements de paramètres et les facteurs sans dimensions qu'elles mettent en évidence, précisent clairement quels effets de couplage sont susceptibles de se produire.

On voit, sur la formule (4.2), que l'effet de couplage sur $\left(q_{u}-q\right)$ est à rechercher entre $\left(C_{0}+q \operatorname{tg} \Phi\right)$ et $(g+\gamma \operatorname{tg} \Phi) B$.

II est important, disposant de la valeur exacte $\mathrm{q}_{\mathrm{u}}$, de la comparer à son évaluation par défaut donnée par la méthode de superposition qui ne tient pas compte de l'effet de couplage ci-dessus : formules (3.7) et (3.9). Remarquons que, puisque la formule (4.2) contient comme cas particulier le cas classique de la semelle filante sur sol illimité homogène, la comparaison proposée permettra d'étalonner le caractère conservatif de la méthode de superposition telle qu'elle est couramment utilisée.

Pour effectuer cette comparaison nous introduisons le coefficient $\mu_{c^{\prime}}$ fonction de $\left.\frac{(\mathrm{g}+\gamma \operatorname{tg} \Phi}{\mathrm{C}_{0}+q \operatorname{tg} \Phi}, \frac{\mathrm{B}}{\mathrm{h}}, \Phi\right)$ défini par :

$$
\mu_{c}=\frac{q_{u}-q}{\left(q_{u}\right)_{\text {superp }}-q}
$$




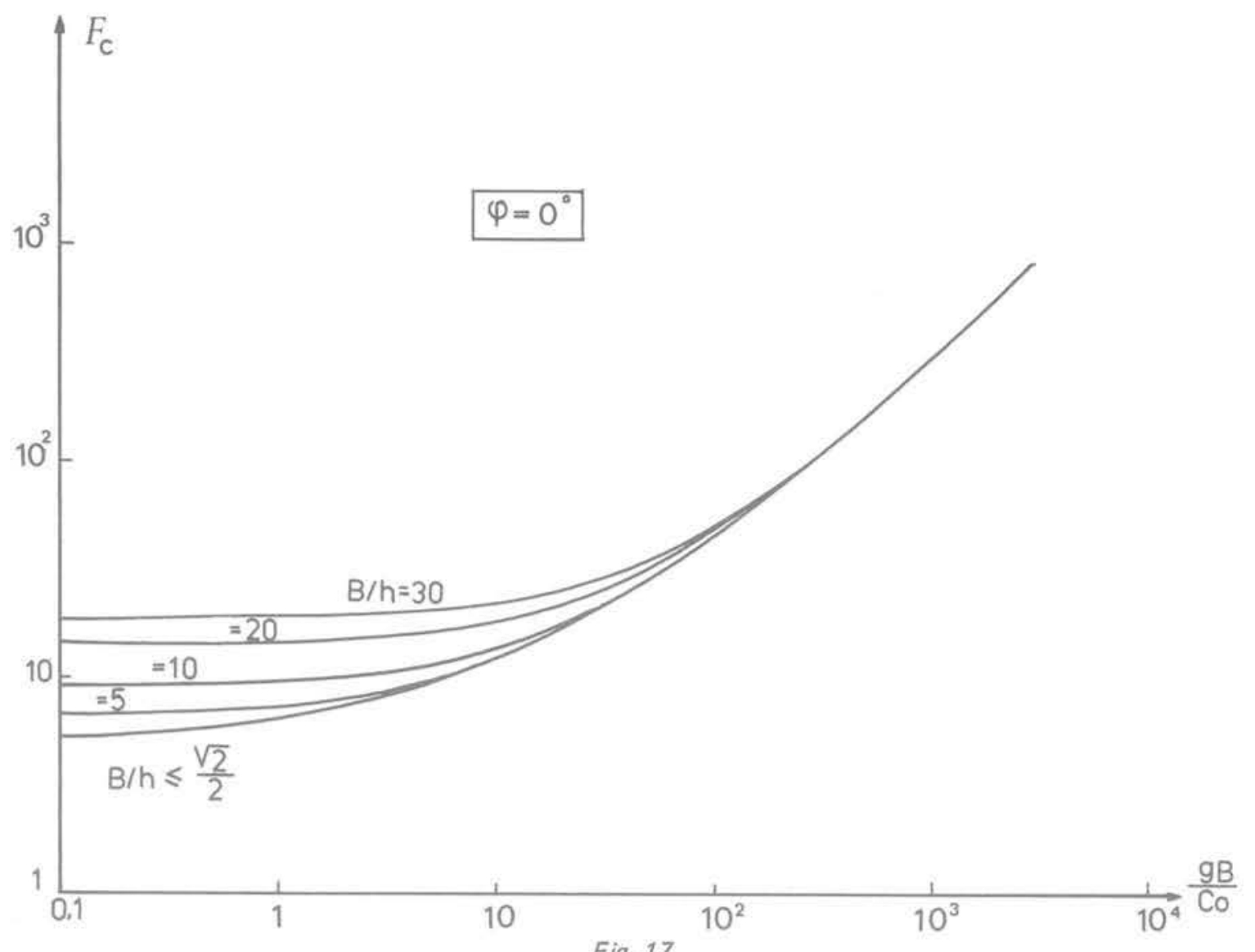

Fig. 17

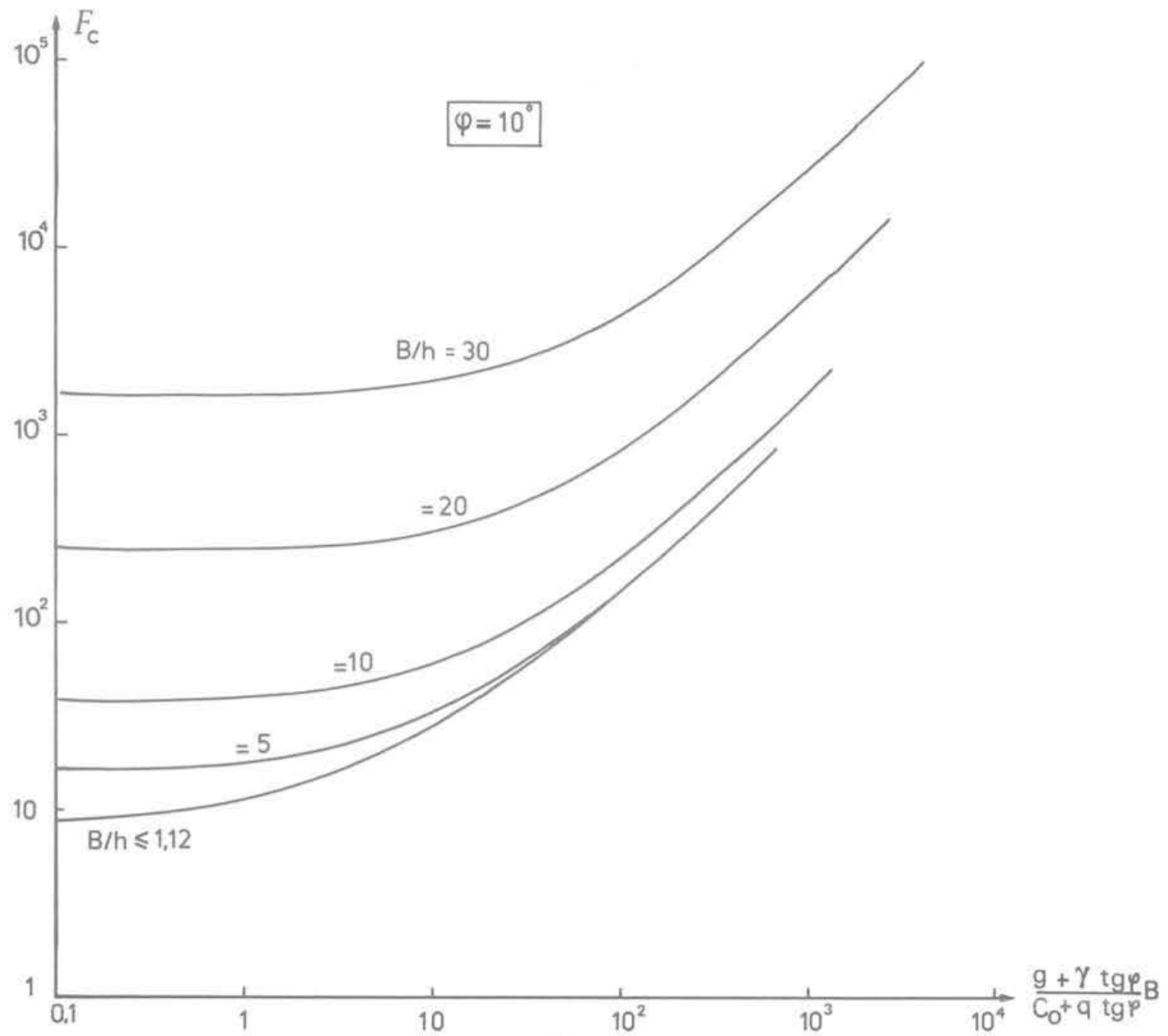

Fig. 18 


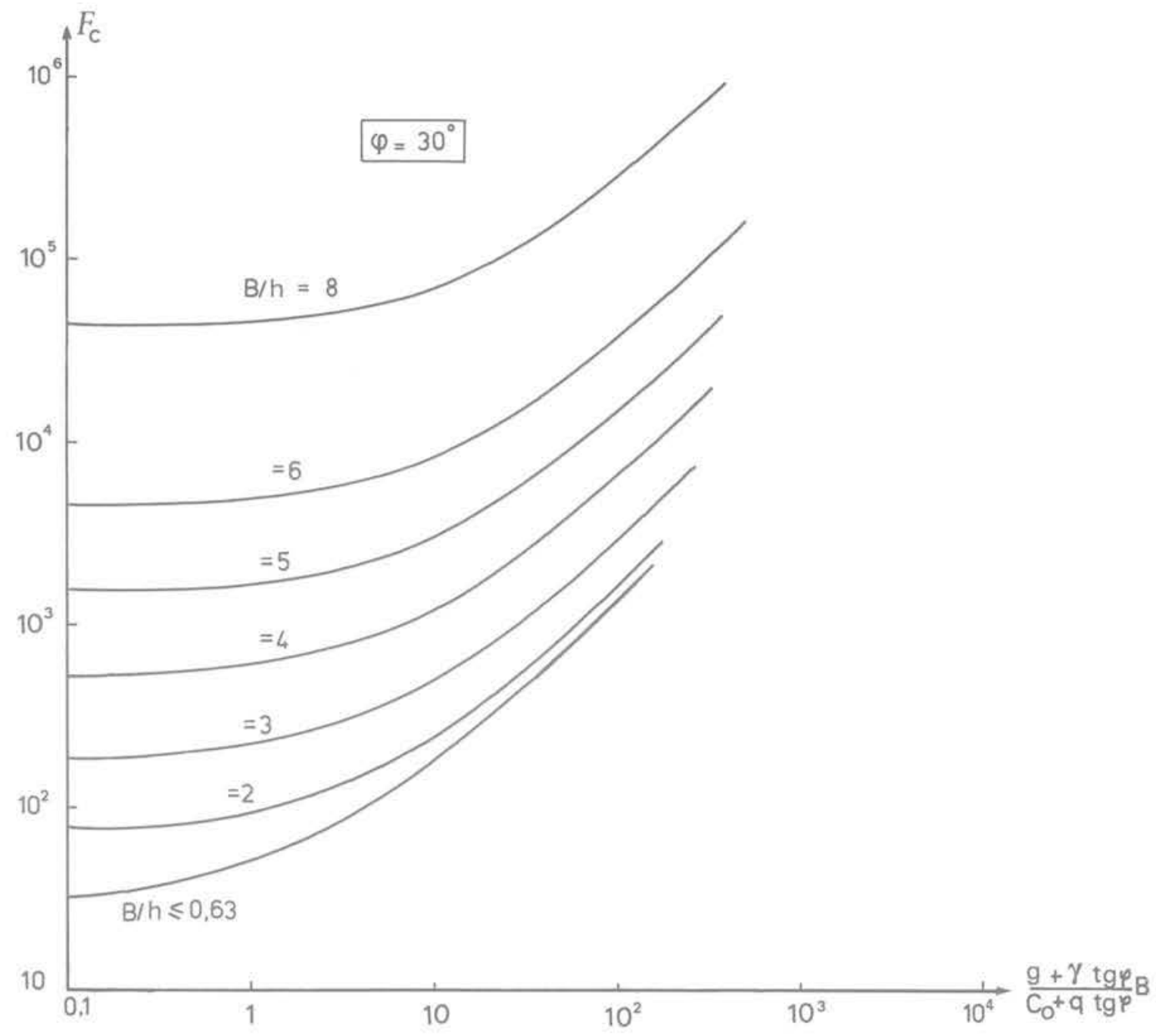

Fig. 19

Ce coefficient s'apparente au coefficient employé par Lundgren et Mortensen (1953).

II représente pour $q=0$, le rapport entre $q_{u}$ et $\left(q_{u}\right)$ superp.

Pour $q \neq 0$, l'introduction de ce coefficient se justifie, en plus de sa commodité pour la représentation des résultats, par le fait que dans la pratique, lorsque l'on procède à un calcul de dimensionnement de fondation, on fait porter le coefficient de sécurité, non sur $\mathrm{q}_{u}$, mais sur $\left(\mathrm{q}_{u}-\mathrm{q}\right)$ ), (cf. Leonards (1968), etc.).

En se reportant aux formules (3.7), (3.9) et (4.2) on obtient pour $\mu_{c}$ :

(4.10)

$$
\text { pour }\left(C_{0}+q \operatorname{tg} \Phi\right) \neq 0 \text {. }
$$

$$
\begin{aligned}
& \mu_{c}\left(\frac{g+\gamma \operatorname{tg} \Phi}{C_{o}+q \operatorname{tg} \Phi} B, \frac{B}{h}, \Phi\right)= \\
& F_{c}\left(\frac{g+\gamma \operatorname{tg} \Phi}{C_{o}+q \operatorname{tg} \Phi} B, \frac{B}{h}, \Phi\right)
\end{aligned}
$$

$\frac{1}{2} \frac{\mathrm{g}+\gamma \operatorname{tg} \Phi}{\mathrm{C}_{\mathrm{o}}+\mathrm{qtg} \Phi} \mathrm{BN}^{\prime}{ }_{\gamma}\left(\frac{\mathrm{B}}{\mathrm{h}}, \Phi\right) \operatorname{cotg} \Phi+\mathrm{N}_{\mathrm{c}}\left(\frac{\mathrm{B}}{\mathrm{h}}, \Phi\right)$ en posant $\left.\mathrm{N}_{\gamma} \cdot \frac{\mathrm{B}}{\mathrm{h}}, \Phi\right) \operatorname{cotg} \Phi=1 / 2$ pour $\Phi=0$.

On a évidemment :

(4.11) $\mu_{c} \geqslant 1 \quad \forall \frac{g+\gamma \operatorname{tg} \Phi}{C_{0}+q \operatorname{tg} \Phi} B, \frac{B}{h}, \Phi \geqslant 0$

D'autre part on peut obtenir une majoration de $\mu_{c}$ en remarquant que $q^{2}$ est nécessairement inférieure d'après le théorème statique du $\$ 2.3$ à la capacité portante d'une semelle sur une couche homogène de cohésion $C_{0}+q \operatorname{tg} \Phi$ $+(g+\gamma \operatorname{tg} \Phi) h$, qui est la cohésion maximale dans la couche pour le problème $\mathrm{P}^{2}$. $\mathrm{D}^{\prime}$ où :

$1 \leqslant \mu_{c}\left(\frac{g+\gamma \operatorname{tg} \Phi}{C_{0}+q \operatorname{tg} \Phi} B, \frac{B}{h}, \Phi\right) \leqslant \frac{1+\frac{g+\gamma \operatorname{tg} \Phi}{C_{0}+q \operatorname{tg} \Phi} B \cdot\left(\frac{B}{h}\right)^{-1}}{\frac{1}{2} \frac{g+\gamma \operatorname{tg} \Phi}{C_{0}+q \operatorname{tg} \Phi} B \frac{N_{\gamma}^{\prime}(B / h, \Phi)}{N_{c}^{\prime}(B / h, \Phi)} \operatorname{cotg} \Phi+1}$ 
Indiquons maintenant les propriétés de $\mu_{\mathrm{c}}$ qui sont démontrées dans (Matar, 1978)

(4.12) $\mu_{\mathrm{c}}\left(0, \frac{\mathrm{B}}{\mathrm{h}}, \Phi\right)=1$ $\forall \frac{\mathrm{B}}{\mathrm{h}}, \forall \Phi \geqslant 0$

(4.13) $\mu_{c}\left(\infty, \frac{\mathrm{B}}{\mathrm{h}}, \Phi\right)=1$ $\forall \frac{\mathrm{B}}{\mathrm{h}}, \forall \Phi \geqslant 0$

$$
\mu_{c}\left(\frac{g+\gamma \operatorname{tg} \Phi}{C_{0}+q \operatorname{tg} \Phi} B, \infty, \Phi\right)=1 \quad \forall \frac{g+\gamma \operatorname{tg} \Phi}{C_{0}+q \operatorname{tg} \Phi} B, \forall \Phi \geqslant 0
$$

Comme indiqué au paragraphe précédent et sur la figure 14 , $(B / H)_{0}$ est une fonction croissante de $\frac{g+\gamma \operatorname{tg} \Phi}{C_{0}+q \operatorname{tg} \Phi} B$ :

$\left(\frac{\mathrm{B}}{\mathrm{h}}\right)_{c} \leqslant\left(\frac{\mathrm{B}}{\mathrm{h}}\right)_{0} \leqslant\left(\frac{\mathrm{B}}{\mathrm{h}}\right)_{\gamma}$

D'oủ, en se reportant à (3.3), on voit immédiatement que :

(4.15) $\left\{\begin{array}{l}\mathrm{Si}(\mathrm{B} / \mathrm{h}) \leqslant(\mathrm{B} / \mathrm{h})_{\mathrm{c}} \\ \text { Alors } \mu_{\mathrm{c}} \text { est indépendant de } \mathrm{B} / \mathrm{h}, \forall \Phi \geqslant 0\end{array}\right.$

par contre, $\mu$ dépend explicitement de $B / h, \forall \Phi \geqslant 0$, dès que $B / h$ est supérieur à $(B / h)$

Ces propriétés, qui seront utilisées pour la constitution des abaques du chapitre 5 , sont résumées sur la figure 20 .

Sur les figures 21,22 et 23 on a représenté $\mu_{\text {en fonction }}$ de son premier argument, par des courbes « iso- $B / h$ \# à $\Phi$ fixé ; sur les figures 24 à 26 on a représenté $\mu$ en fonction de son premier argument par des courbes « iso- $\Phi$ " aे $\mathrm{B} / \mathrm{h}$ fixe pour les petites valeurs de $\mathrm{B} / \mathrm{h}\left({ }^{1}\right)$. On constate que l'on obtient des courbes en cloches qui appellent les commentaires suivants :

Pour les figures 21 à 23 on voit que :

- la valeur maximale $\mu_{c} \max$ de $\mu_{c}$ correspondant au pic des courbes, est une fonction décroissante de $B / h$;

- la sous-estimation de la capacité portante introduite par l'utilisation de la méthode de superposition peut être importante, en particulier pour les petites valeurs de $\mathrm{B} / \mathrm{h}$.

Pour les figures 24 à 26 ;

- la valeur maximale $\mu_{c} \max$ de $\mu_{c}$ correspondant au pic des courbes, est une fonction décroissante de $\Phi$ :

- la sous-estimation de la capacité portante introduite par l'utilisation de la méthode de superposition peut être importante, en particulier pour les petites valeurs de $\Phi$.

Pour un sol purement cohérent $(\Phi=0), \mu_{c}$ passe par un maximum égal à 1,72 pour $\mathrm{gB} / \mathrm{C}_{0}=22$.

II ressort de ces résultats que la sous-estimation de la capacité portante introduite par l'utilisation de la méthode de superposition peut être importante, en particulier pour les petites valeurs de $\mathrm{B} / \mathrm{h}$ et de $\Phi$, et va jusqu'à atteindre $40 \%$ de la valeur exacte de $\mathrm{q}_{4}$

\section{Abaques pour le calcul pratique de la capacité portante des semelles filantes}

\subsection{Présentation}

Les grandes valeurs numériques atteintes par la capacité portante $q_{u}$ imposent de représenter celle-ci graphique-

(1) Le cas $\mathrm{B} / \mathrm{h}=0$ correspondant au sol illimité avait été donné par Salencon. Florentin et Gabriel (1976).

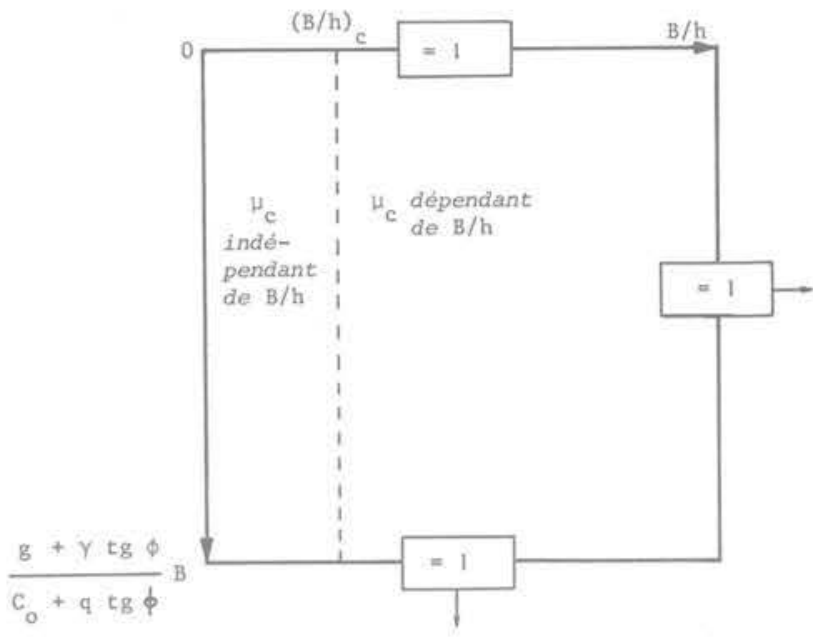

Fig. 20 Propriétés de $\mu_{c}$

ment à l'aide d'une échelle logarithmique comme nous l'avons fait sur les figures 17 à 19 pour $F_{\text {. }}$. Ce type de représentation n'est pas utilisable commodément dans la pratique.

L'utilisation de la méthode de superposition étant habituellement assez aisée, nous proposons de fonder le calcul pratique de la capacité portante sur cette méthode en employant des coefficients correcteurs, à partir de la définition de $\mu_{c}$ donnée par la formule (4.9).

Pour cela on donne à $\Phi$ fixé, les courbes « iso- $\mu_{c}$ " dans le plan $\left(\frac{\mathrm{g}+\gamma \operatorname{tg} \Phi}{\mathrm{C}_{\mathrm{o}}+\mathrm{q} \operatorname{tg} \Phi} \mathrm{B}, \frac{\mathrm{B}}{\mathrm{h}}\right)$ déjà introduit à la figure 20.

En regard de ce plan, on a tracé les courbes donnant $\mathrm{N}^{\prime}{ }_{c}(\mathrm{~B} / \mathrm{h}, \Phi)$ et $\mathrm{N}_{\gamma}(\mathrm{B} / \mathrm{h}, \Phi) \operatorname{cotg} \Phi$ en fonction de $\mathrm{B} / \mathrm{h}$.

Ces abaques, où les valeurs des coefficients $\mu_{c^{\prime}} N^{*}{ }_{c^{\prime}}$ $\mathrm{N}^{\prime} \gamma \operatorname{cotg} \Phi$ peuvent être lues ou interpolées, sont d'un emploi commode pour calculer dans la pratique la capacité portante, en appliquant les formules suivantes déduites de (3.7), (3.9) et (4.9):

Pour un sol de Coulomb $(\Phi \neq 0)$

$\mathrm{q}_{\mathrm{u}}=\mathrm{q}+\mu_{\mathrm{c}}\left(\mathrm{C}_{\mathrm{o}}+\mathrm{q} \operatorname{tg} \Phi\right)\left[\frac{1}{2} \frac{\mathrm{g}+\gamma \operatorname{tg} \Phi}{\mathrm{C}_{\mathrm{o}}+\mathrm{q} \operatorname{tg} \Phi} \mathrm{B} \mathrm{N}_{\gamma}^{\prime} \operatorname{cotg} \Phi+\mathrm{N}_{\mathrm{c}}\right]$

Pour un sol purement cohérent $(\Phi=0)$

$$
\mathrm{q}_{u}=\mathrm{q}+\mu_{\mathrm{c}} \mathrm{C}_{0}\left(\mathrm{~N}_{\mathrm{c}}^{\prime}+\frac{1}{4} \frac{\mathrm{gB}}{\mathrm{C}_{0}}\right)
$$

Nous donnons dans la suite les abaques " iso- $\mu_{\mathrm{c}}$ " correspondant à $\Phi=0^{\circ}, 4^{\circ}, 10^{\circ}, 20^{\circ}, 25^{\circ}, 30^{\circ}, 35^{\circ}, 40^{\circ}$ et $45^{\circ}$.

5.2 Abaques « iso- $\mu_{c}$ » pour

$\Phi=0^{\circ}, 4^{\circ}, 10^{\circ}, 20^{\circ}, 25^{\circ}, 30^{\circ}, 35^{\circ}, 40^{\circ}, 45^{\circ}$. 


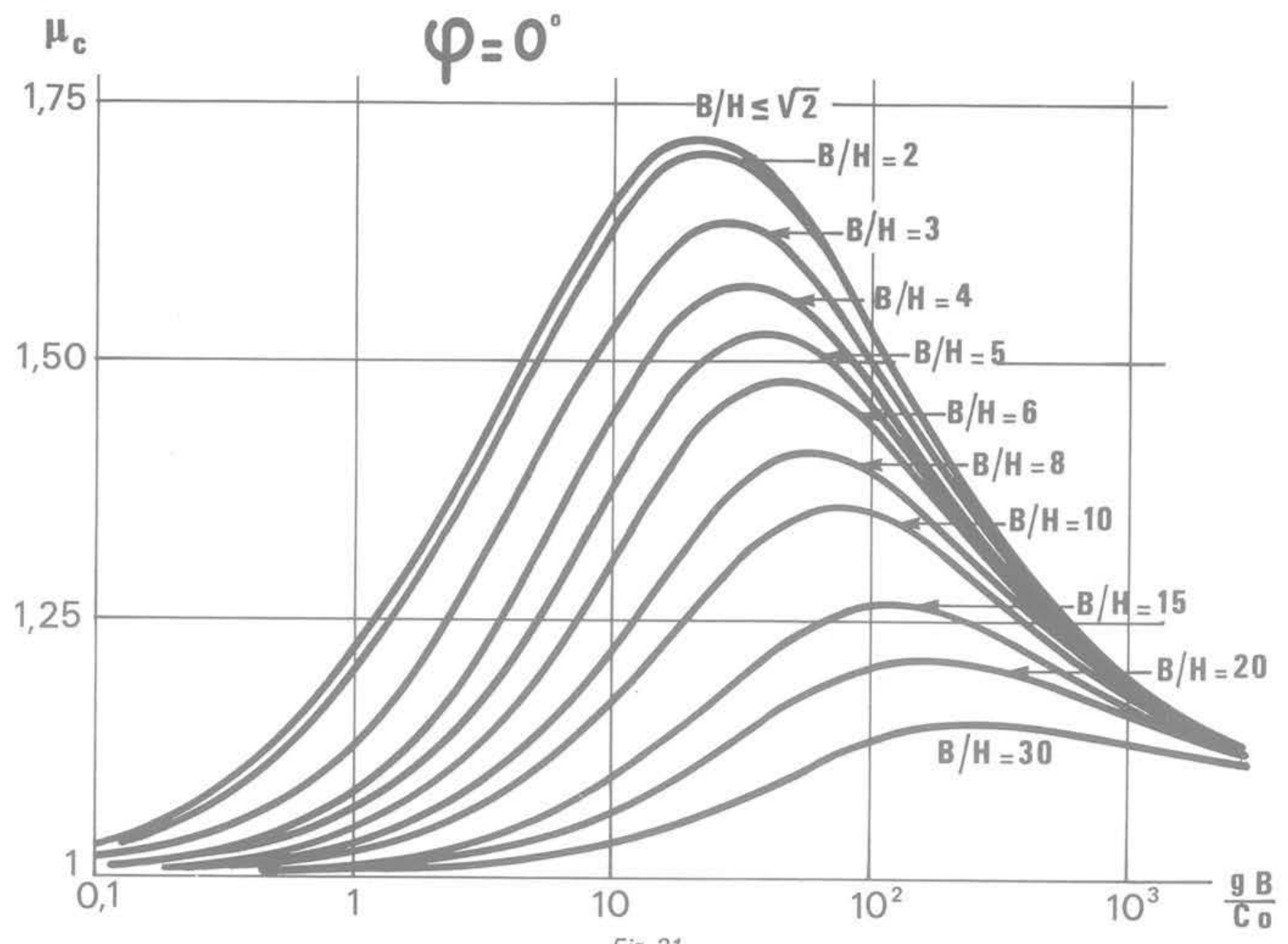

Fig. 21

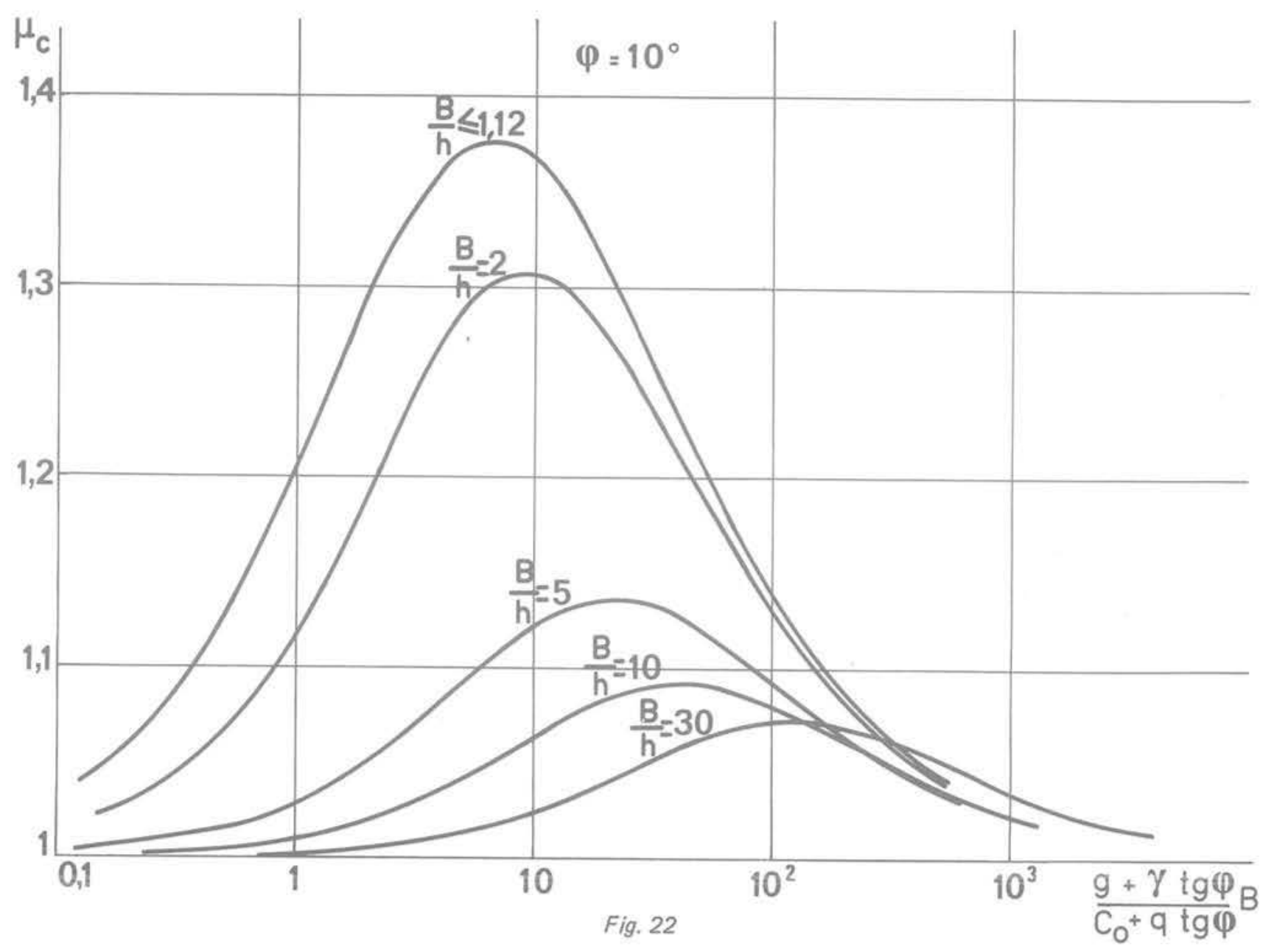




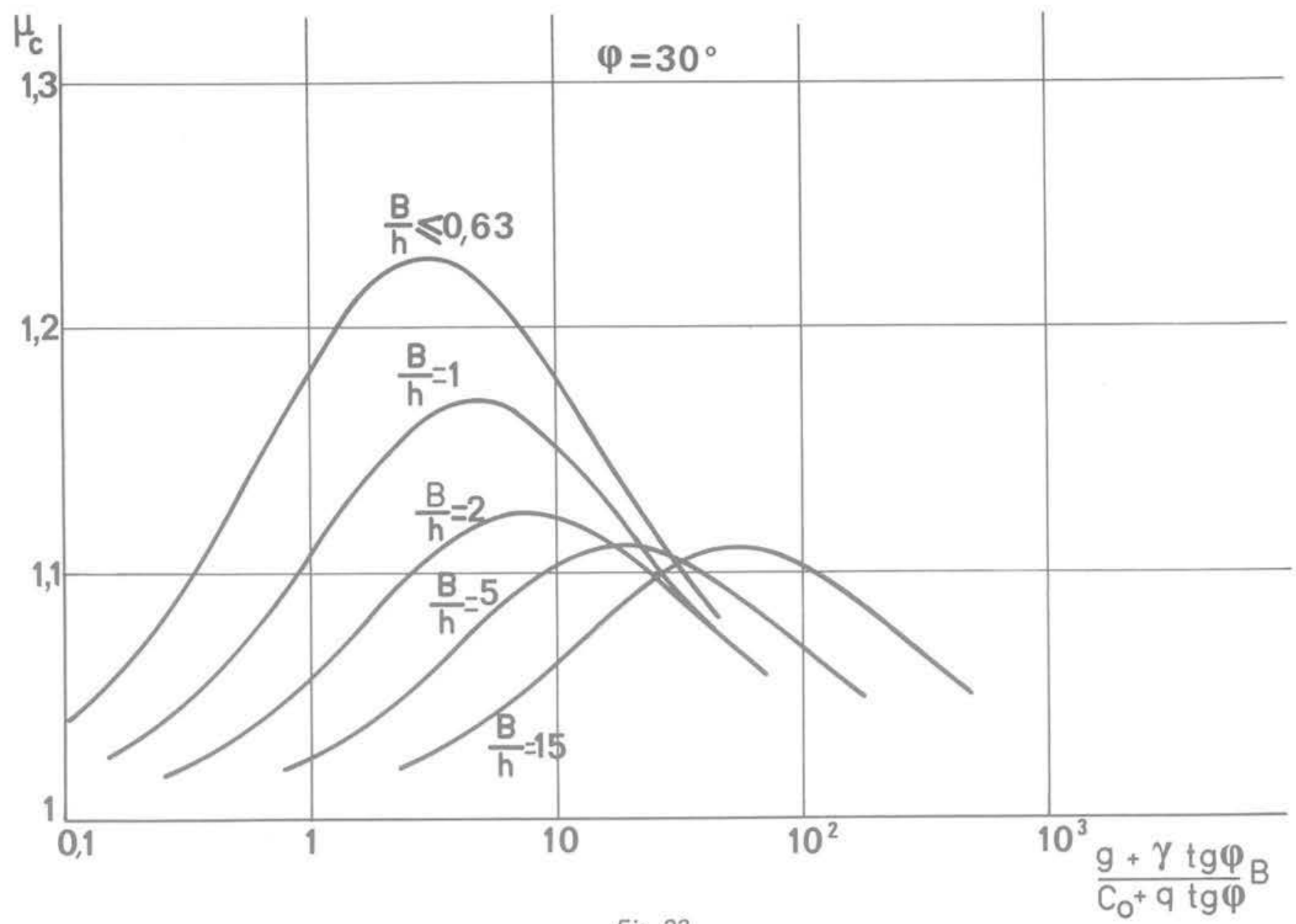

Fig. 23

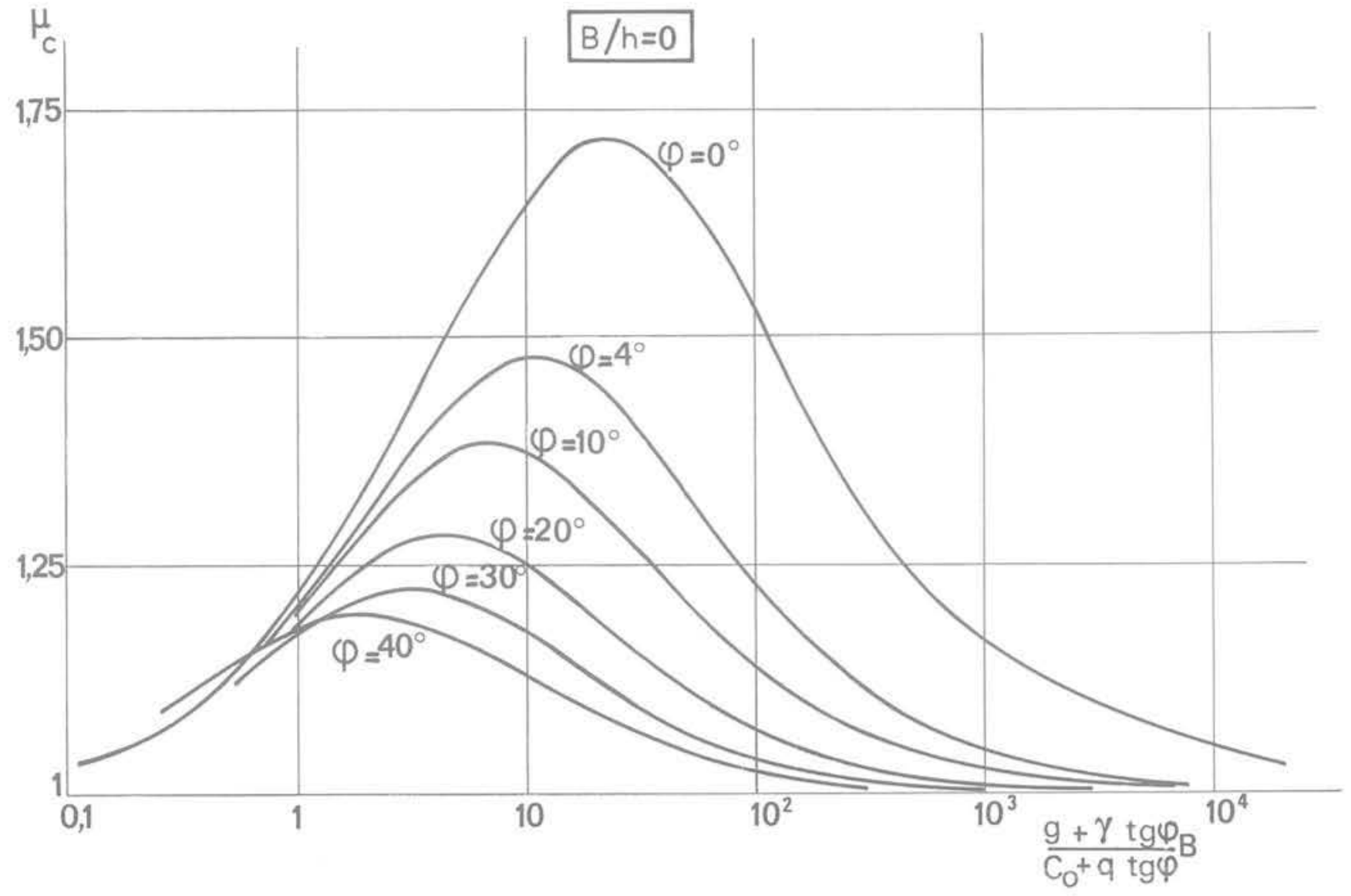

Fig. 24 


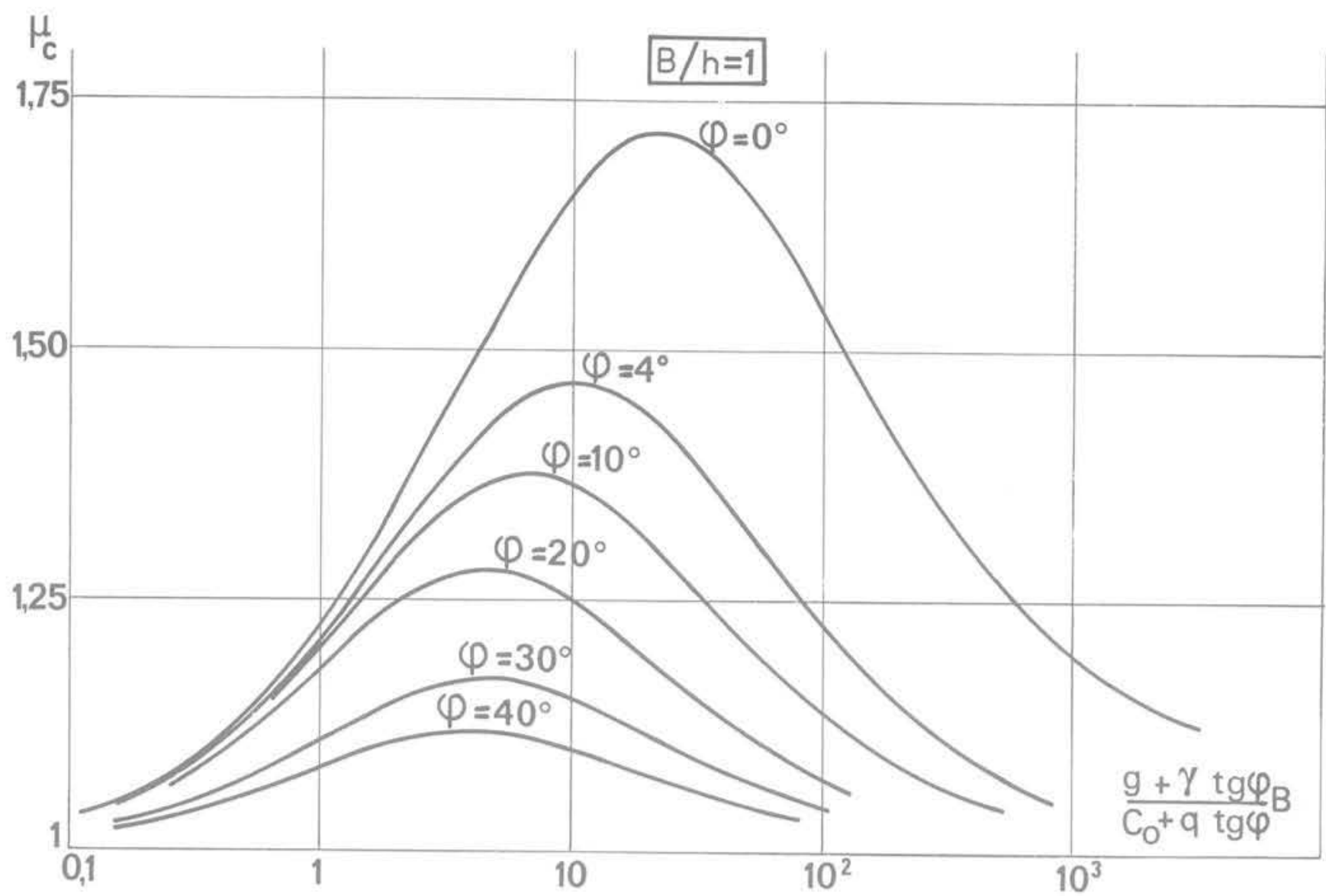

Fig. 25

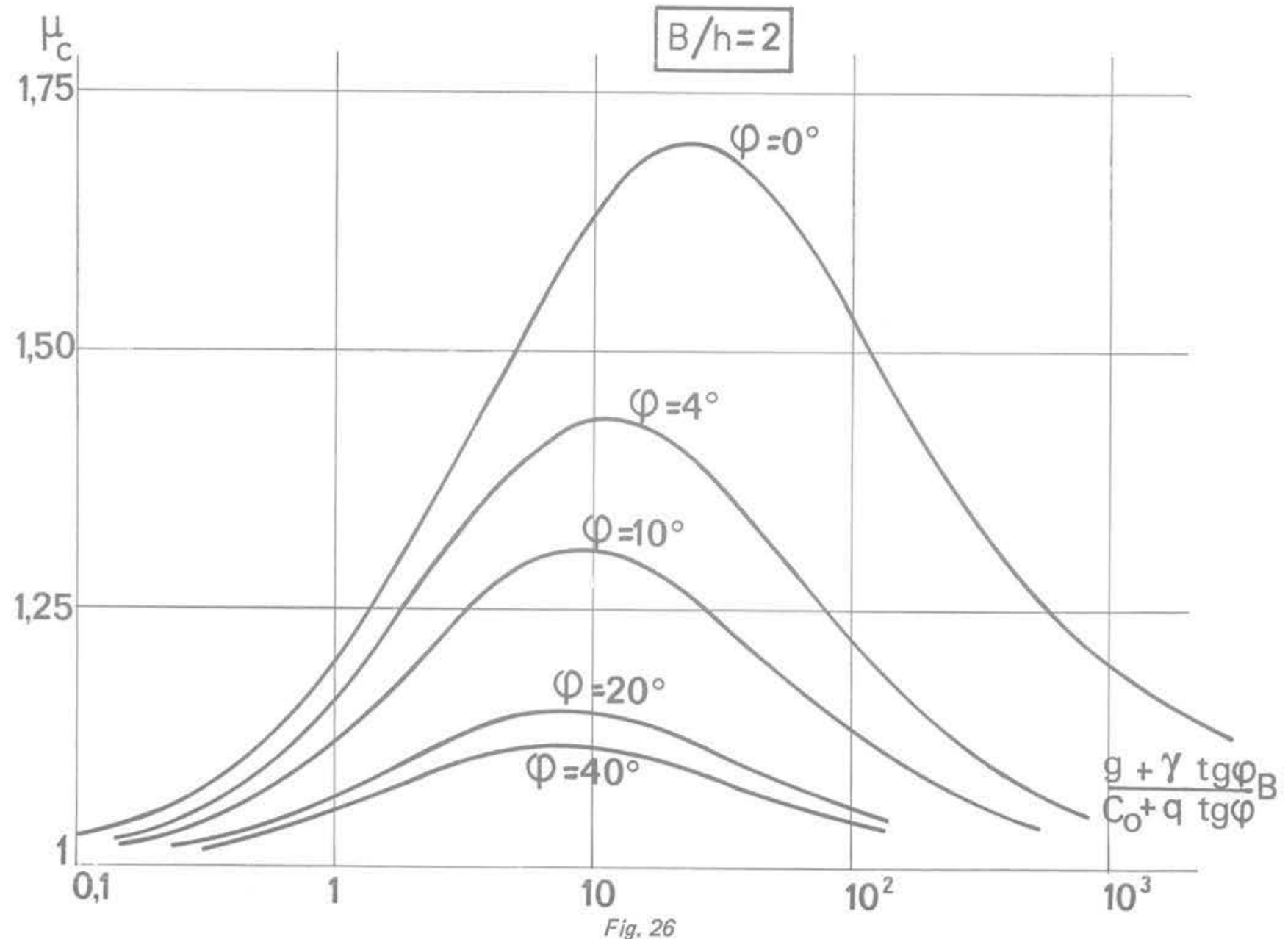




\section{Exemples d'utilisation des abaques « iso- $\mu_{c}$ "}

\subsection{Présentation}

On trouvera dans la référence (Matar, 1978) des exemples très détaillés concernant l'application de la méthode de superpostion et du calcul global, ainsi que de diverses "règles » pratiques utilisées dans le cas d'une semelle filante reposant sur une couche de sol non homogène d'épaisseur limitée. Pour ne pas alourdir l'exposé nous nous restreindrons à n'en présenter ici que les plus significatifs.

\subsection{Premier exemple ; site A.}

Sol d'angle de frottement interne : $\Phi=0^{\circ}$, puis $4^{\circ}, 10^{\circ}$ Largeur de la fondation : $B=40 \mathrm{~m}$, puis $4 \mathrm{~m}$

Surcharge latérale $: q=0$

Epaisseur de la couche

$: \mathrm{h} \doteq 10 \mathrm{~m}$

Cohésion en surface

$: \mathrm{C}_{\mathrm{o}}=10^{3} \mathrm{~Pa}$

Gradient vertical descendant de cohésion

Poids volumique

$\mathrm{g}=2,5 \times 10^{3} \mathrm{~N} / \mathrm{m}^{3}$

$: \gamma=1,6 \times 10^{4} \mathrm{~N} / \mathrm{m}^{3}$

$1^{\circ} \mathrm{B}=40 \mathrm{~m}$

On lit sur les abaques de la figure $27\left(\Phi=0^{\circ}\right)$ :

$$
\mu_{\mathrm{c}}=1,48 \quad \mathrm{~N}_{\mathrm{c}}=6,25
$$

$$
\begin{aligned}
& \text { d'où } \quad\left(\mathrm{q}_{\mathrm{u}}\right)_{\text {superp. }}=3,12 \times 10^{4} \mathrm{~Pa} \\
& \text { et } \quad q_{u} \quad=4,62 \times 10^{4} \mathrm{~Pa}
\end{aligned}
$$

ce qui met en évidence l'importance de la sous-estimation de la capacité portante par la méthode de superpositiion.

Le cas d'une couche de sol non-homogène d'épaisseur limitée, ayant été traité par Mandel et Salençon (1969 et 1972), il arrive que dans la pratique on traite le problème posé ci-dessus comme s'il s'agissait d'une couche de sol homogène, par des règles telles que :

- en ne prenant en compte qu la cohésion en surface, ce qui aboutit à une évaluation par défaut de la capacité portante :

$$
\left(q_{u}\right)_{0}=0,625 \times 10^{4} \mathrm{~Pa}
$$

Cette valeur conduit à des dimensionnements très surabondants.

- en prenant en compte une cohésion "moyenne \# qui pourrait être, par exemple:

$$
C_{m}=C_{0}+g \frac{h}{2}
$$

où h' est l'épaisseur de la zone perturbée correspondant à un sol homogène :

$$
h^{\prime}=\min \left(h, \frac{B \sqrt{2}}{2}\right)
$$

II vient alors :

$$
C_{m}=13,5 \times 10^{3} \mathrm{~Pa}
$$

et la capacité portante estimée est:

$$
\overline{\mathrm{q}}_{\mathrm{u}}=8,44 \times 10^{4} \mathrm{~Pa}
$$

Outre sa difficulté d'emploi, cette méthode conduit à une surestimation de la capacité portante qui est évidemment inacceptable.

Sur la figure 36 , nous dressons une comparaison entre les valeurs de la capacité portante obtenues par les différentes méthodes.

II n'est pas sans intérêt d'examiner l'influence que peut avoir l'angle de frottement, même très petit, sur la valeur de la capacité portante. Sur l'exemple ci-dessus, en considérant $\Phi=0^{\circ}, 4^{\circ}, 10^{\circ}$, nous obtenons :

$$
\begin{array}{ll}
\text { pour } \Phi=0^{\circ} & \mathrm{q}_{u}=4,62 \times 10^{4} \mathrm{~Pa} \\
\text { Pour } \Phi=4^{\circ} & \mathrm{q}_{u}=10,24 \times 10^{4} \mathrm{~Pa} \\
\text { pour } \Phi=10^{\circ} & \mathrm{q}_{u}=29,3 \times 10^{4} \mathrm{~Pa}
\end{array}
$$

ce qui met en évidence (figure 37) l'effet stabilisateur très marqué de l'angle de frottement interne, du point de vue de la capacité portante.

$2^{\circ} \mathrm{B}=4 \mathrm{~m}$

On va voir que les remarques faites ci-dessus sont valables aussi dans le cas d'une semelle filante de largeur plus réduite :

On lit sur la figure 27 :

$$
\mu_{c}=1,65
$$

d'où :

$$
\mathrm{q}_{\mathrm{u}}=1,26 \times 10^{4} \mathrm{~Pa}
$$

L'utilisation des méthodes empiriques pour traiter le cas de la couche non-homogène évoquées plus haut conduit aे :

en prenant

$$
\begin{array}{ll}
\mathrm{C}=\mathrm{C}_{0} & \left(\mathrm{q}_{\mathrm{u}}\right)_{\mathrm{o}}=0,51 \times 10^{4} \mathrm{~Pa} \\
\mathrm{C}=\mathrm{C}_{\mathrm{m}} & \overline{\mathrm{q}_{u}}=2,33 \times 10^{4} \mathrm{~Pa}
\end{array}
$$

La figure 38 présente la comparaison entre les valeurs obtenues par les diverses méthodes.

On peut aussi mettre en évidence l'effet stabilisateur très marqué de l'angle de frottement interne.

\subsection{Deuxième exemple : site $\mathrm{B}\left({ }^{1}\right)$}

Sol d'angle de frottement interne : $\Phi=0^{\circ}$, puis $4^{\circ}, 10^{\circ}$

Largeur de la fondation $\quad: B=40 \mathrm{~m}$

Surcharge latérale $\quad: q=0$

Epaisseur de la couche $\quad: h=\infty$

Cohésion en surface $\quad: \mathrm{C}_{0}=0$

Gradient vertical descendant

de cohósion

$; g=0,6 \times 10^{3} \mathrm{~N} / \mathrm{m}^{3}$

Poids volumique

$: \gamma=1,6 \times 10^{4} \mathrm{~N} / \mathrm{m}^{3}$

Pour le sol purement cohérent $(\Phi=0)$ on obtient par les diverses méthodes:

$$
\begin{aligned}
& q_{u}=\left(q_{u}\right)_{\text {superp. }}=0,6 \times 10^{4} \mathrm{~Pa} \\
& \left(q_{u}\right)_{0}=0 \quad \text { la construction sera impossible. } \\
& \overline{q_{u}}=4,36 \times 10^{4} \mathrm{~Pa} \quad \text { Surestimation inacceptable. }
\end{aligned}
$$

L'effet stabilisateur de l'angle de frottement interne est également très marqué :

$$
\begin{array}{ll}
\Phi=0 & \mathrm{q}_{u}=0,6 \times 10^{4} \mathrm{~Pa} \\
\Phi=4^{\circ} & \mathrm{q}_{u}=3,85 \times 10^{4} \mathrm{~Pa} \\
\Phi=10^{\circ} & \mathrm{q}_{\mathrm{u}}=16,83 \times 10^{4} \mathrm{~Pa}
\end{array}
$$

(1) Argile en Méditerranée (Le Tirant, 1976). 


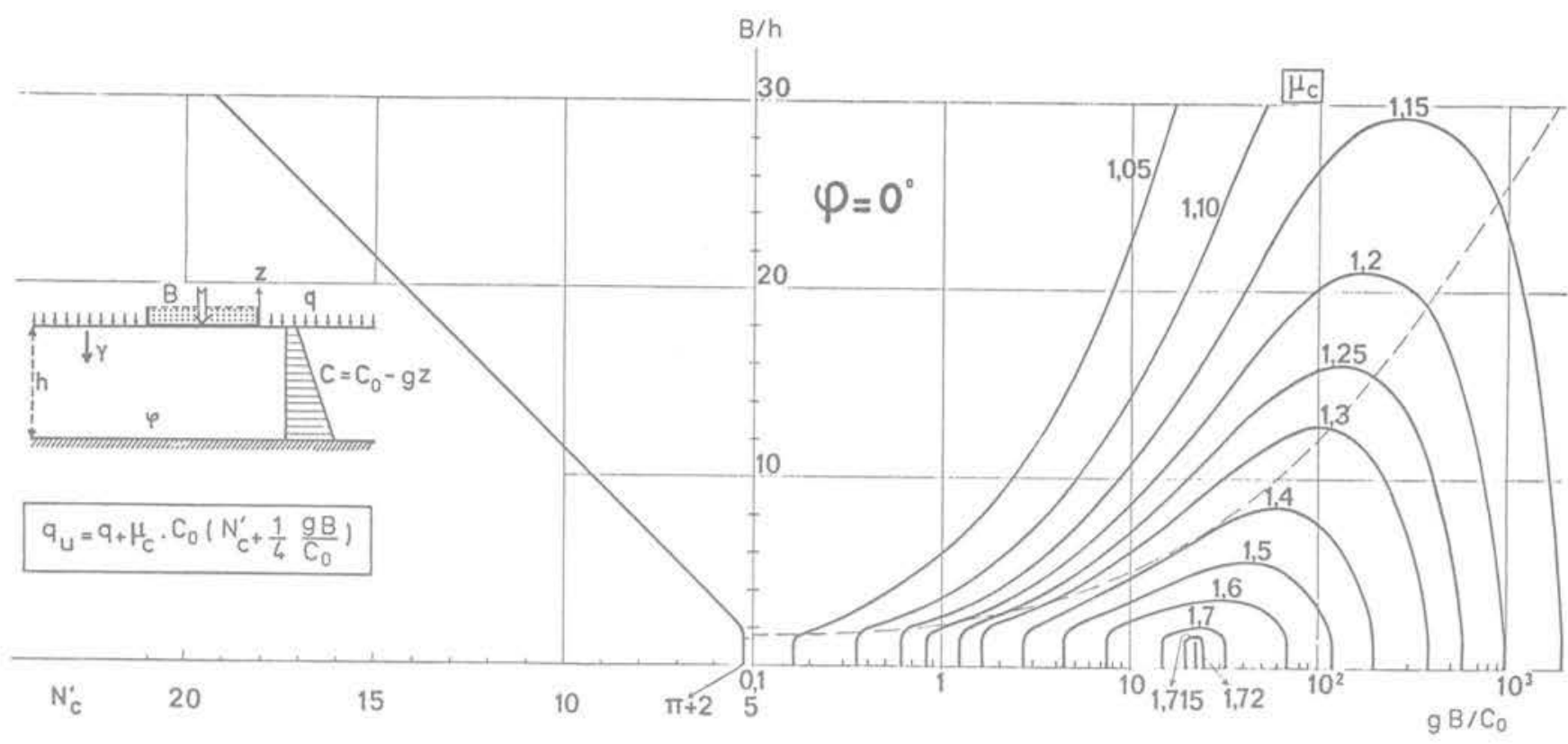

Fig. 27

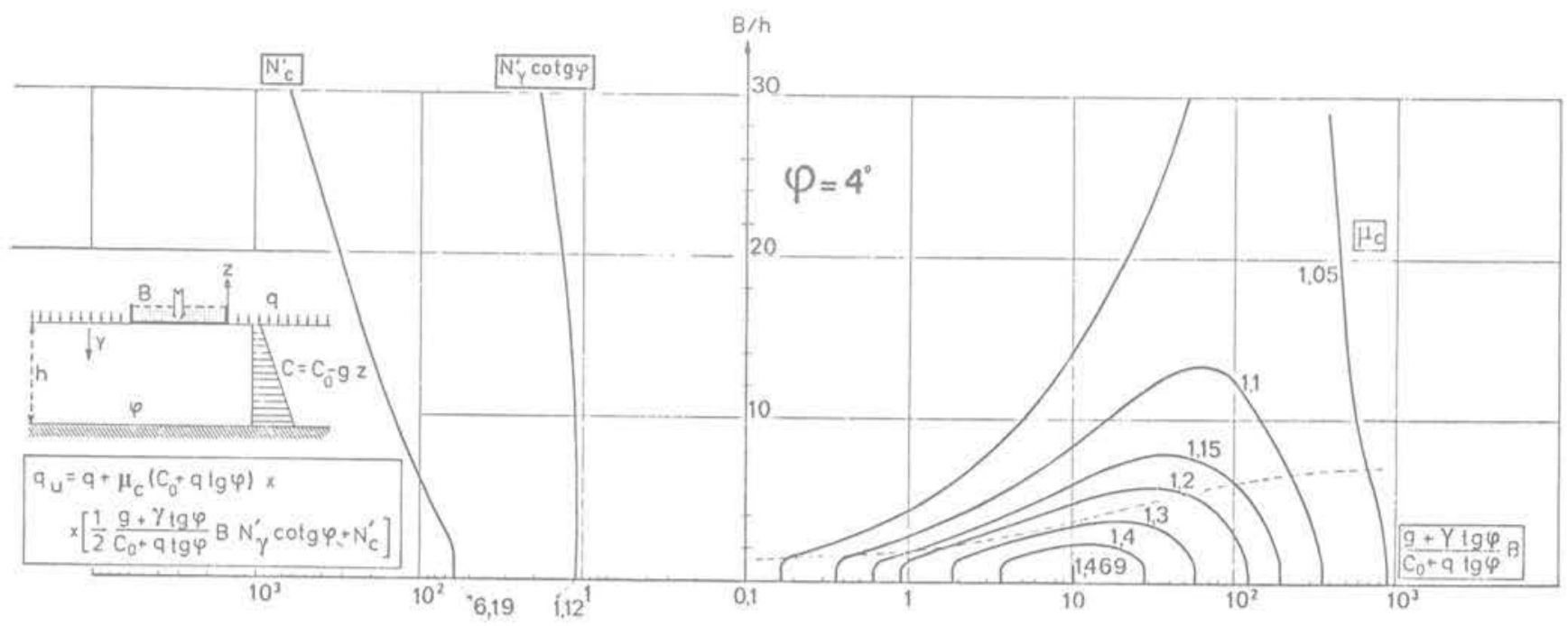

Fig. 28

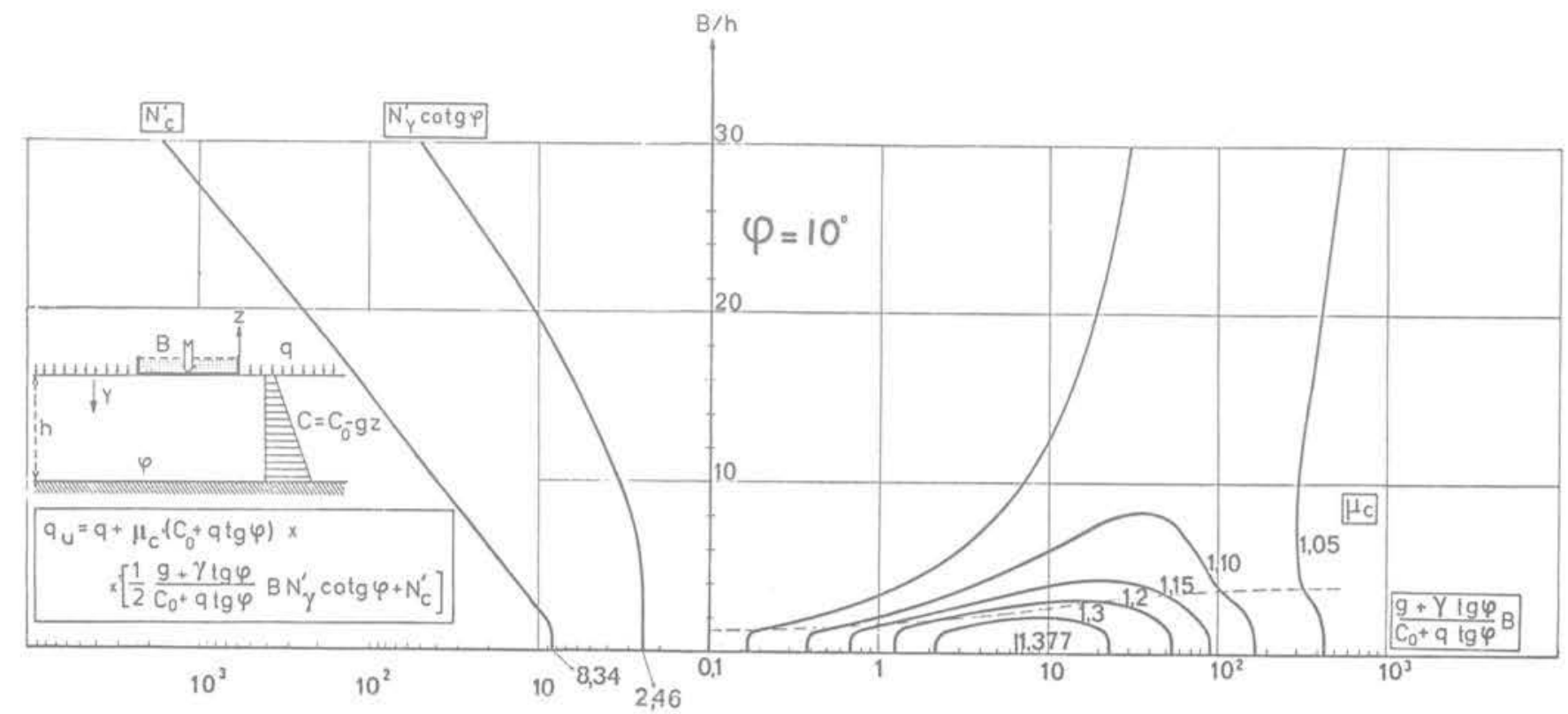

Fig. 29 


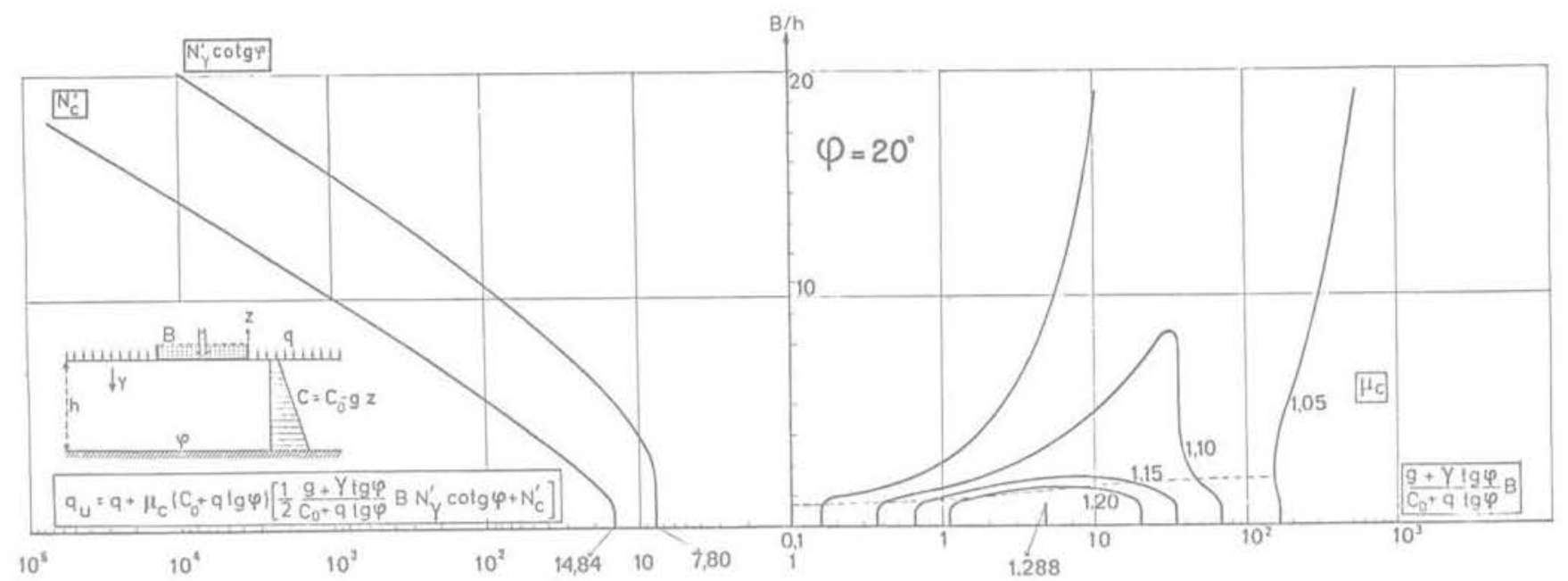

Fig. 30

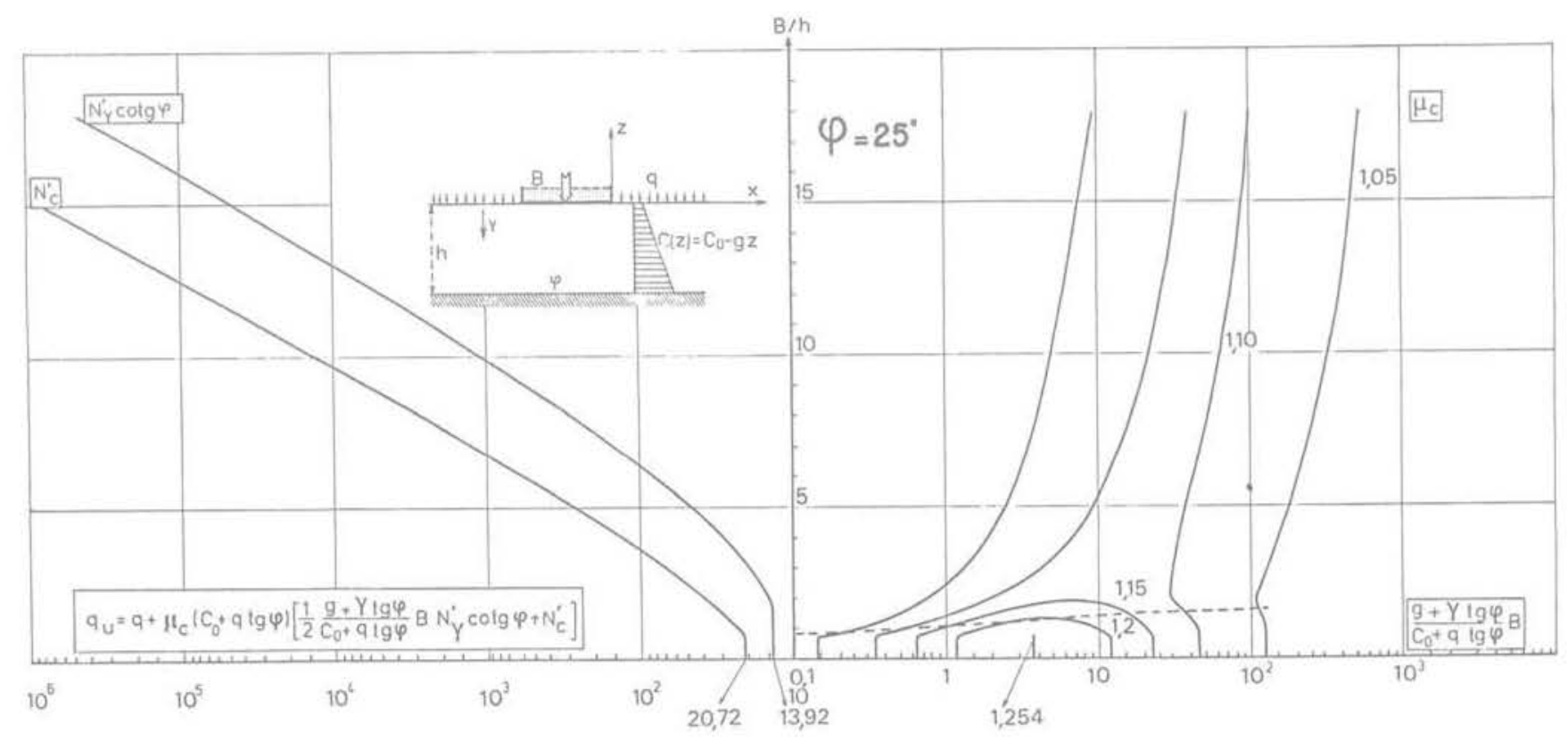

Fig. 31

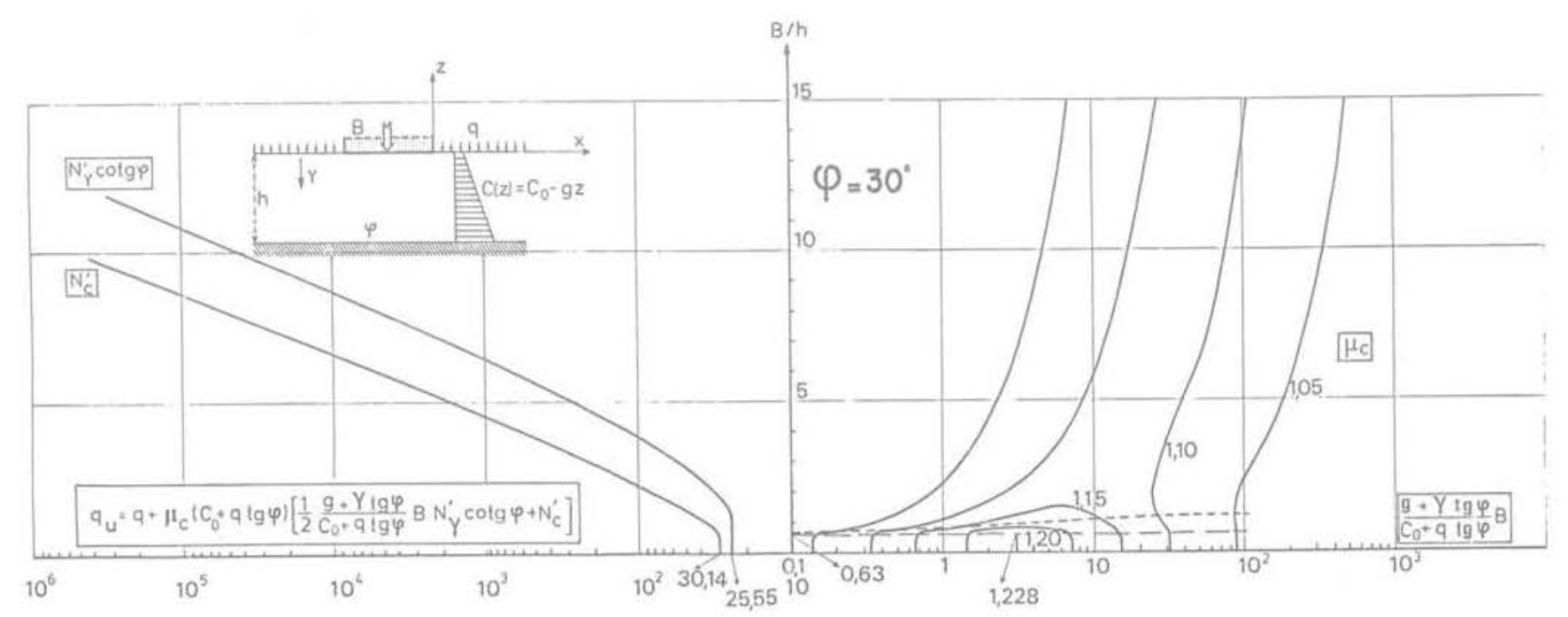

Fig. 32 


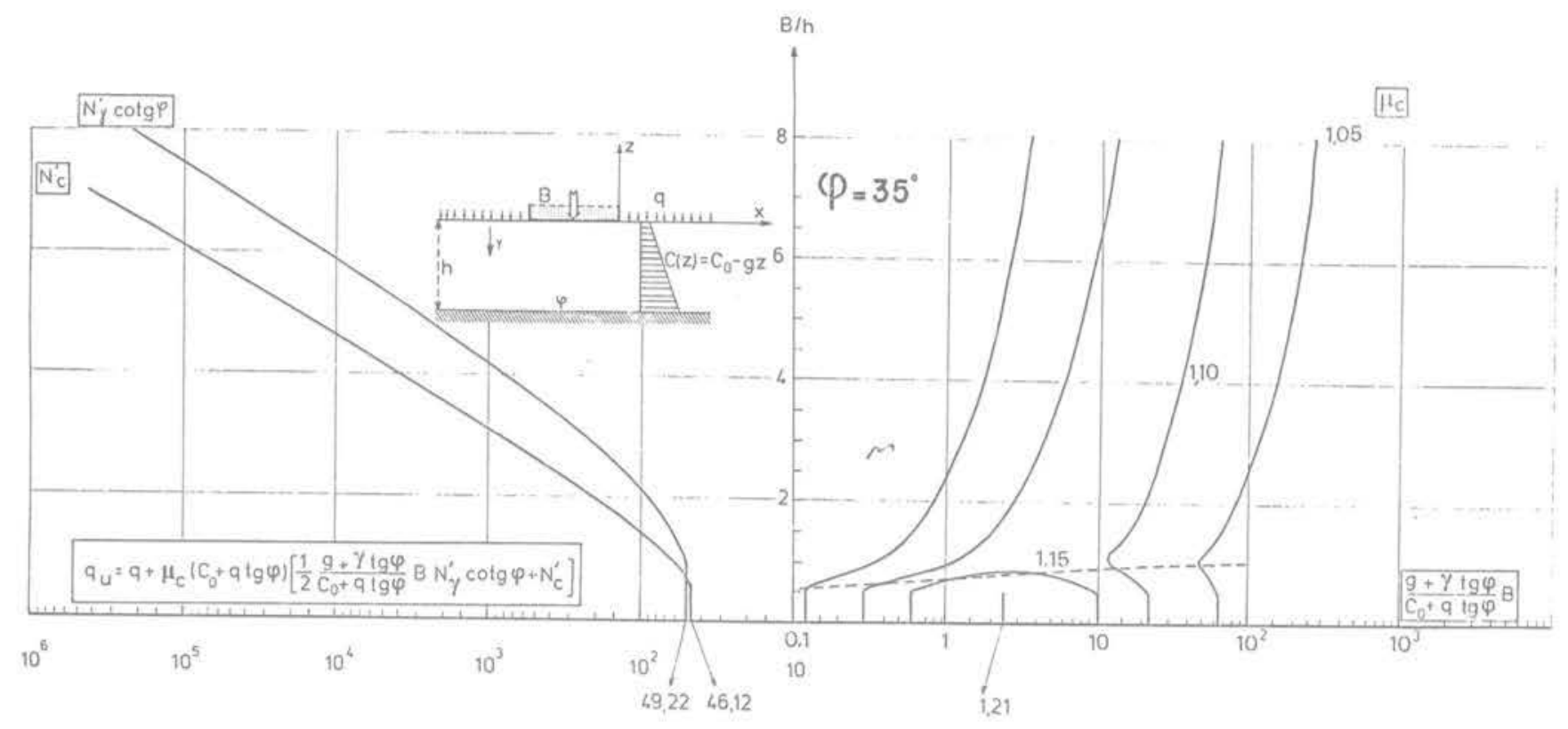

Fig. 33

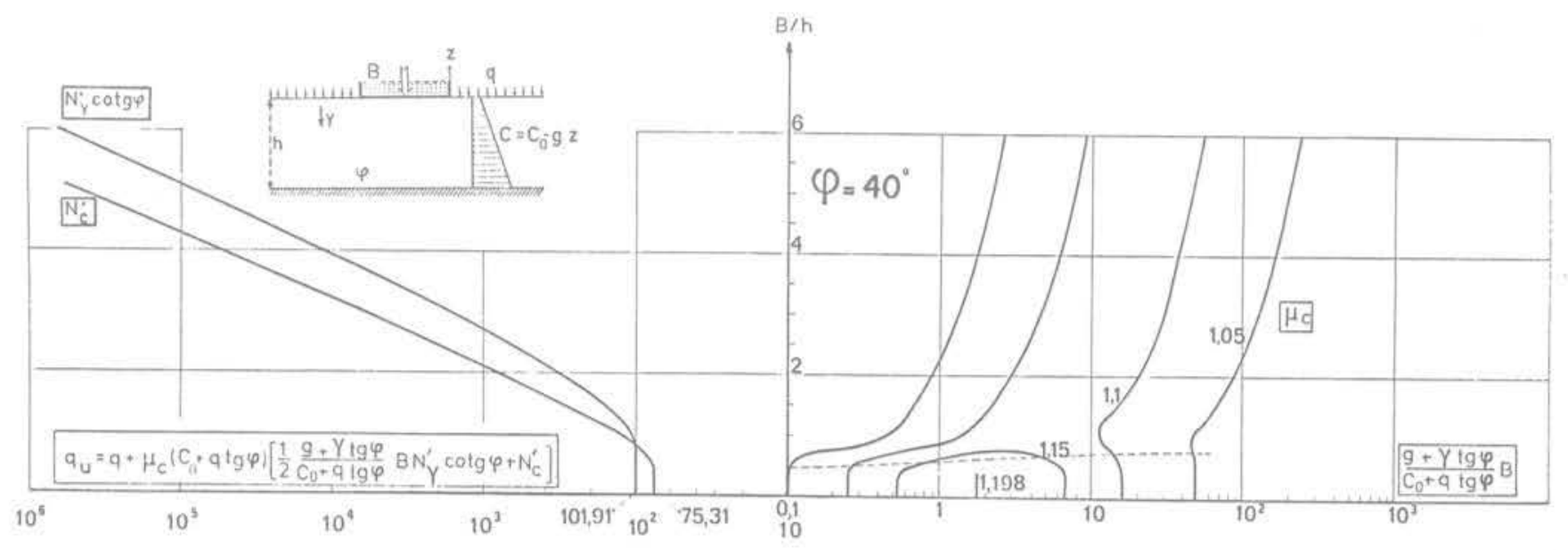

Fig. 34

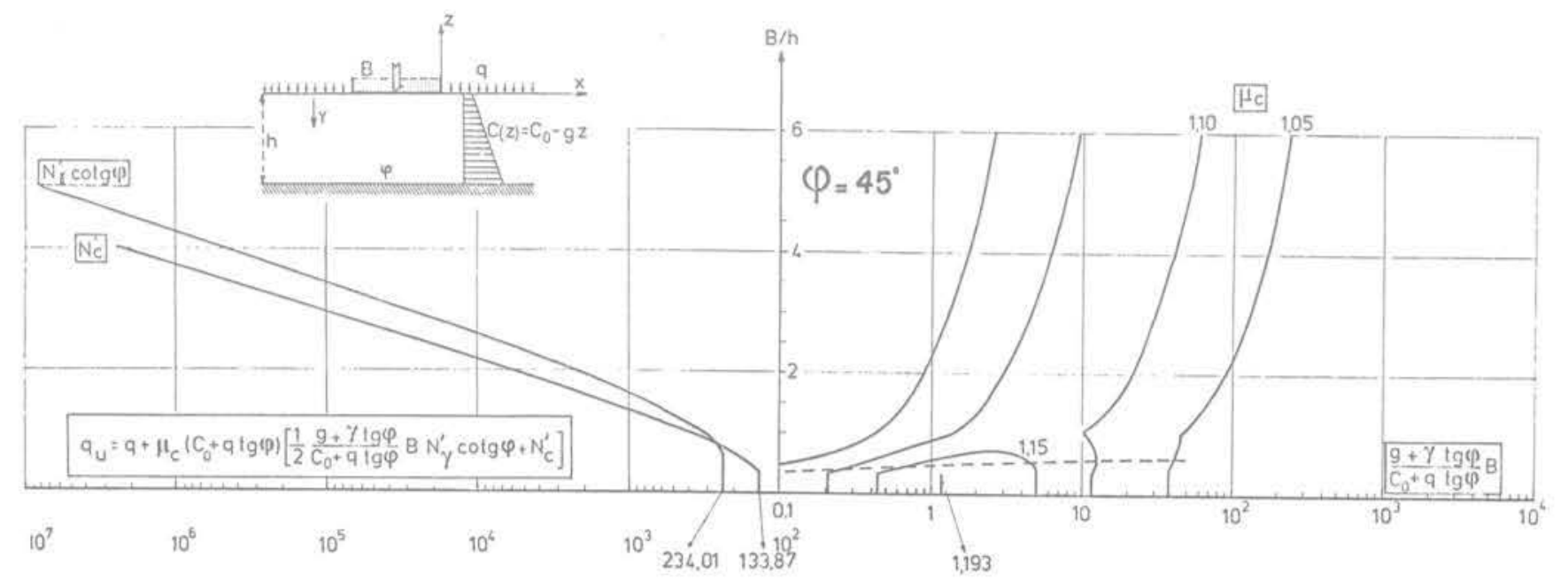

Fig. 35 


$$
\mathrm{q}_{\mathrm{u}} / 10^{4} \mathrm{~Pa}
$$

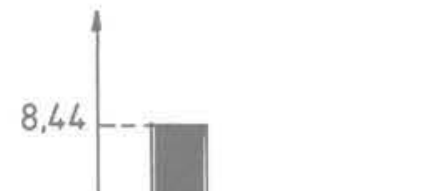

3,12

0.63
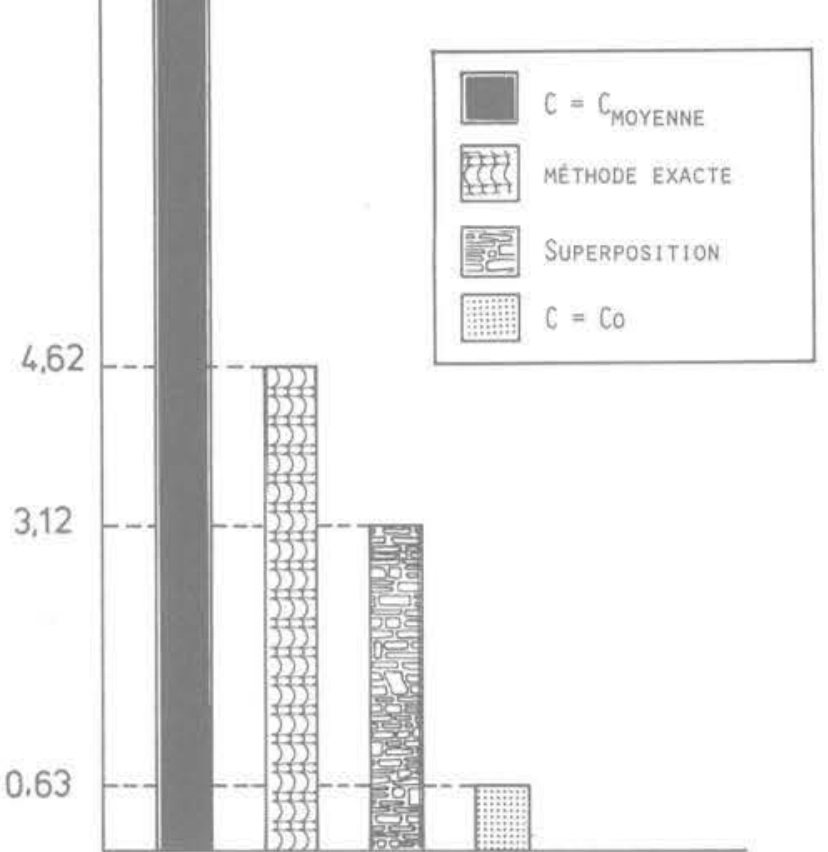

Fig. 36

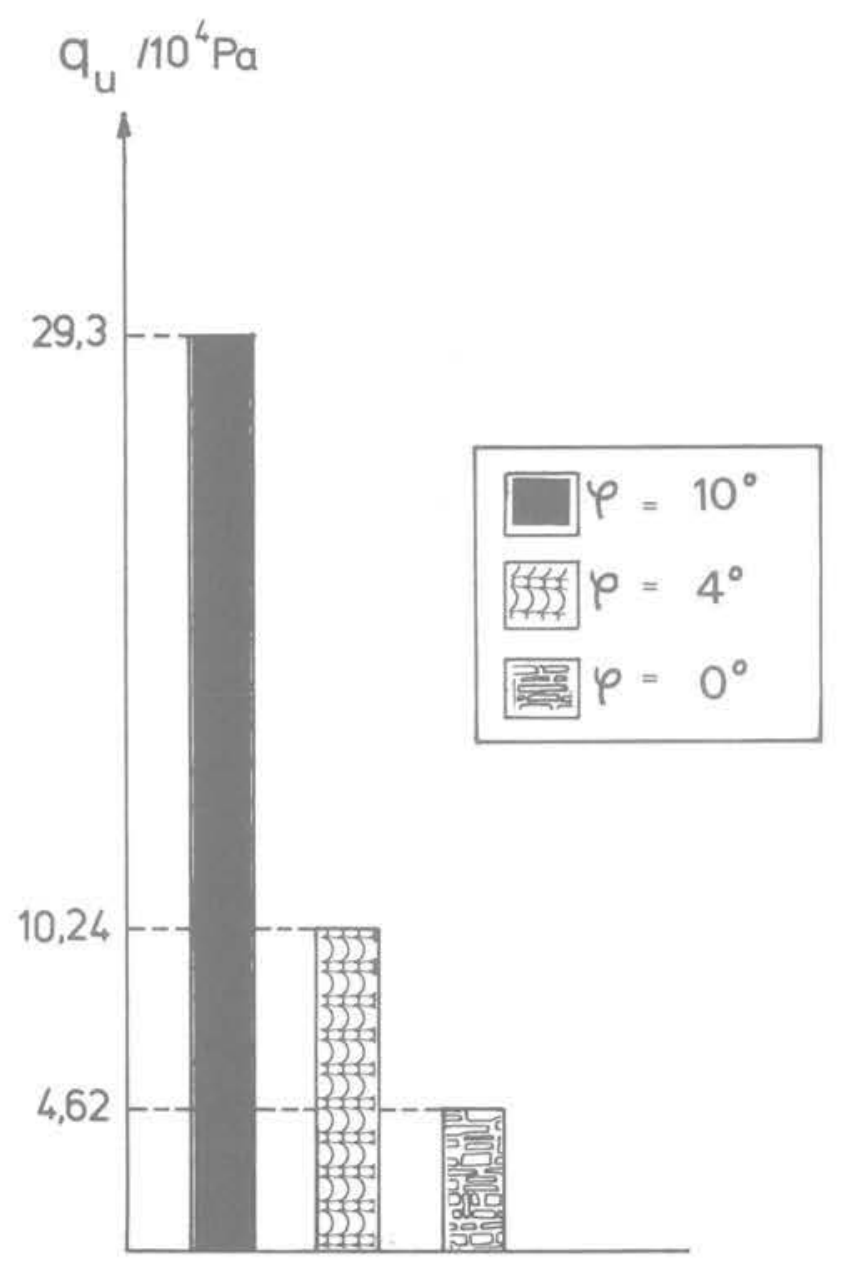

Fig. 37

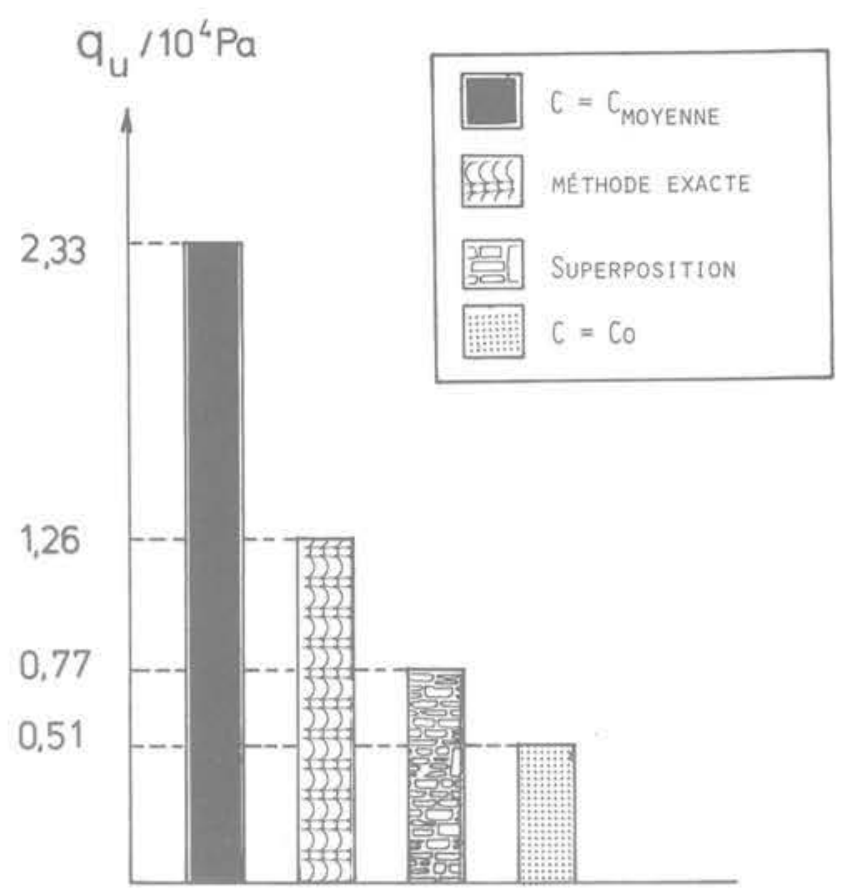

Fig. 38

\subsection{Troisième exemple : site $C$}

Sol frottant

$\Phi=30^{\circ}$

Largeur de la fondation

: $\mathrm{B}=4 \mathrm{~m}$

Poids volumique

$: \gamma=1,8 \times 10^{4} \mathrm{~N} / \mathrm{m}^{3}$

Surcharge latérale

(profondeur de la semelle ; $1 \mathrm{~m}$ )

$: q=1,8 \times 10^{4} \mathrm{~Pa}$

Epaisseur de la couche

$: h=\infty$

Cohésion de surface

$: \mathrm{C}_{0}=1,6 \times 10^{4} \mathrm{~Pa}$

Gradient vertical descendant

de cohésion (milieu homogène) : $\mathrm{g}=0$

En se reportant aux abaques de la figure 32 , on lit:

d'où :

$$
\begin{aligned}
& \mu_{c}=1,20 \\
& q_{u}=1,20\left(q_{u}\right)_{\text {superp. }}-0,20 q ;
\end{aligned}
$$

la sous-estimation de la capacité portante due à l'utilisation de la méthode de superposition dans ce cas est de l'ordre de $20 \%$.

\section{Comparaison avec les résultats d'essais sur modèles}

\subsection{Présentation}

Le but de la comparaison entre les résultats de l'étude théorique présentée dans les chapitres ci-dessus, concrétisés par les abaques pour le calcul de la capacité portante des semelles filantes, et les résultats d'essais sur modèles a été exposé aux chapitres 1 et 2 (\$ 2.6). II s'agit d'apprécier la portée pratique, vis-à-vis du problème modélisé, de la notion de capacité portante telle qu'elle a été définie. 
La figure 39 représente schématiquement un dispositif d'essais dans lequel on devra tenter de satisfaire toutes les conditions pour reproduire le problème modélisé, en particulier du point de vue de la semelle filante; citons par exemple :

- la fondation est rigide et chargée axialement avec une densité linéique constante :

- l'adhérence est totale entre la fondation et le sol :

- le bac d'essais est rigide ;

- l'adhérence est totale entre le fond du bac d'essais et le sol :

- les faces latérales du bac d'essai, perpendiculaires à l'axe longitudinal de la fondation, sont lisses et en contact direct avec la fondation :

- les faces latérales parallèles à l'axe longitudinal sont suffisamment loin pour ne pas gêner le développement du mécanisme de rupture. De plus, il faut qu'elles soient à adhérence totale avec le sol.

\subsection{Résultats et commentaires}

Dans cette comparaison on se réfère aux essais réalisés par Tournier (1) pour l'étude de la capacité portante d'une semelle filante sur une couche de sol homogène d'épaisseur limitée. Ils ont été effectués sur une couche de sable sec d'épaisseur $h$, de poids volumique moyen $\gamma \simeq 1,64 x$ $10^{4} \mathrm{~N} / \mathrm{m}^{3}$ et d'angle de frottement interne $\Phi=38^{\circ}$ (déterminé au triaxial cylindrique). Plusieurs valeurs de $\mathrm{B} / \mathrm{h}$ ont été étudiées pour des semelles de largeur $\mathrm{B}=0,20 \mathrm{~m}$, $0,35 \mathrm{~m}$ et $0,50 \mathrm{~m}$.

Le problème modélisé est donc l'un des problèmes de base pour l'étude de la capacité portante d'une semelle sur une couche de sol non homogène: c'est le problème a) du $\S 3.1$, correspondant à la capacité portante théorique donnée par (3.1 a) :

$$
\mathrm{q}_{u}=\frac{1}{2} \gamma \mathrm{BN}_{\gamma}^{\prime}(\mathrm{B} / \mathrm{h}, \Phi)
$$

Le tableau 2 présente les valeurs déterminées expérimentalement pour le rapport $2 q_{/} / \gamma B$, c'est-à-dire le $" \mathrm{~N}_{\gamma}^{\prime}$ expérimental ».

\begin{tabular}{|c|c|c|c|}
\hline $\mathrm{B} / \mathrm{h}$ & $0,20 \mathrm{~m}$ & $0,35 \mathrm{~m}$ & $0,50 \mathrm{~m}$ \\
\hline 0,15 & 150 & & \\
\hline 0,33 & 150 & 128 & 96 \\
\hline 0,5 & 150 & 128 & 101 \\
\hline 0,67 & & & 114 \\
\hline 0,8 & 184 & & \\
\hline 1 & 231 & 124 & 134 \\
\hline 1,16 & & 194 & \\
\hline 1.41 & & 244 & \\
\hline 2 & 508 & 192 & \\
\hline
\end{tabular}

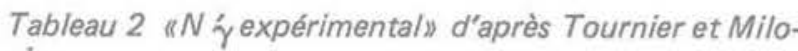
vic

(1) Tournier (1972), Tournier et Milovic (1977).

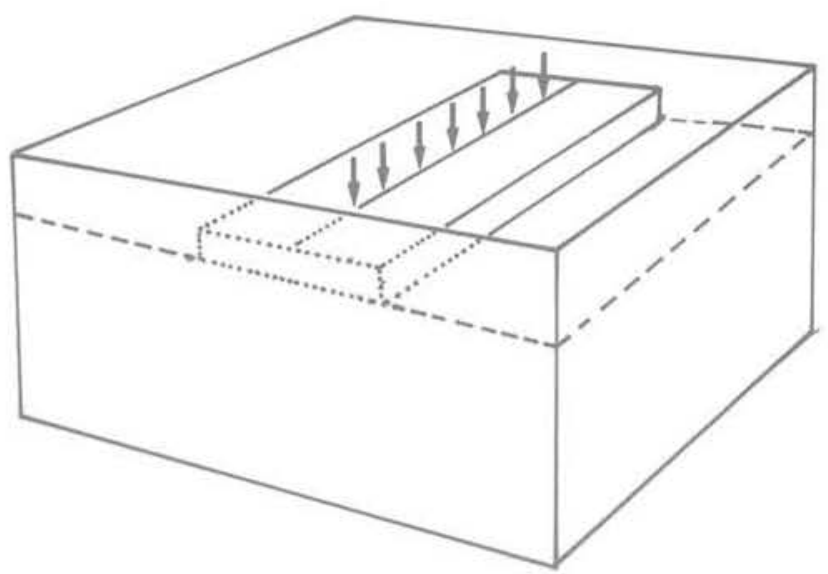

Fig. 39 Bac d'essais

Ces résultats mettent clairement en évidence les difficultés expérimentales (divergence des valeurs obtenues pour une même valeur de $\mathrm{B} / \mathrm{h}$ par exemple); diverses causes peuvent être évoquées, dont la moindre n'est pas la difficulté d'apprécier la rupture de la fondation et donc de décider de la valeur de la charge à prendre en compte !

Ils sont représentés sur la figure 40 où l'on remarque que les valeurs trouvées sont toutes supérieures à la courbe théorique correspondant à $\Phi=38^{\circ}$. Diverses explications peuvent être avancées :

- la rupture de la fondation ne se produit pas dans la géométrie initiale, mais après un certain tassement qui conduit pour la capacité portante théorique à utiliser, non plus la formule (3.1 a), mais la formule globale $(5.1)$ pour tenir compte de la surcharge :

- les parois de la cuve d'essais peuvent ne pas être parfaitement lisses:

- un écrouissage du sol sous la fondation peut aussi être évoqué.

On a tracé, sur la figure 40 , une courbe pointillée qui, laissant de côté un point aberrant, représenterait assez bien les résultats expérimentaux. On constate que cette courbe serait en bonne concordance avec la courbe représentative de $\mathrm{N}_{\gamma}(\underline{\mathrm{B}}, \Phi)$ à condition de prendre pour $\Phi$ la valeur $42^{\circ}$.

Cela peut conduire à mettre en cause la valeur de l'angle de frottement interne à utiliser pour les calculs relatifs aux semelles filantes, c'est-à-dire en fait à adopter pour le sol un critère de rupture tridimensionnel autre qu'un critère " courbe-intrinsèque " de Coulomb: on trouvera dans (Salençon et Halphen, 1979) des éléments de discussion sur ce point qui se rattache aux expériences au « vrai triaxial „ réalisées par de nombreuses équipes (par ex. Lade et Duncan, 1973) et aux réflexions de Habib (1958 et 1961$)$.

La conclusion qu'il nous paraît important de retenir de cette comparaison est que la valeur théorique exacte de la capacité portante issue de la définition du § 2.2 et calculée par la formule théorique (3.1 a) avec les coefficients indiqués en utilisant l'angle de frottement relevé au triaxial est inférieure à la charge qui apparaît expérimentalement comme correspondant à la rupture de la fondation. Ce résultat va dans le sens de la sécurité; il serait intéressant de le confirmer par des essais dans des conditions où l'effet de couplage se manifesterait, q étant alors calculée par (5.1) ou (5.2), notamment pour les cas où $\mu_{c}$ atteint ses valeurs les plus importantes. 


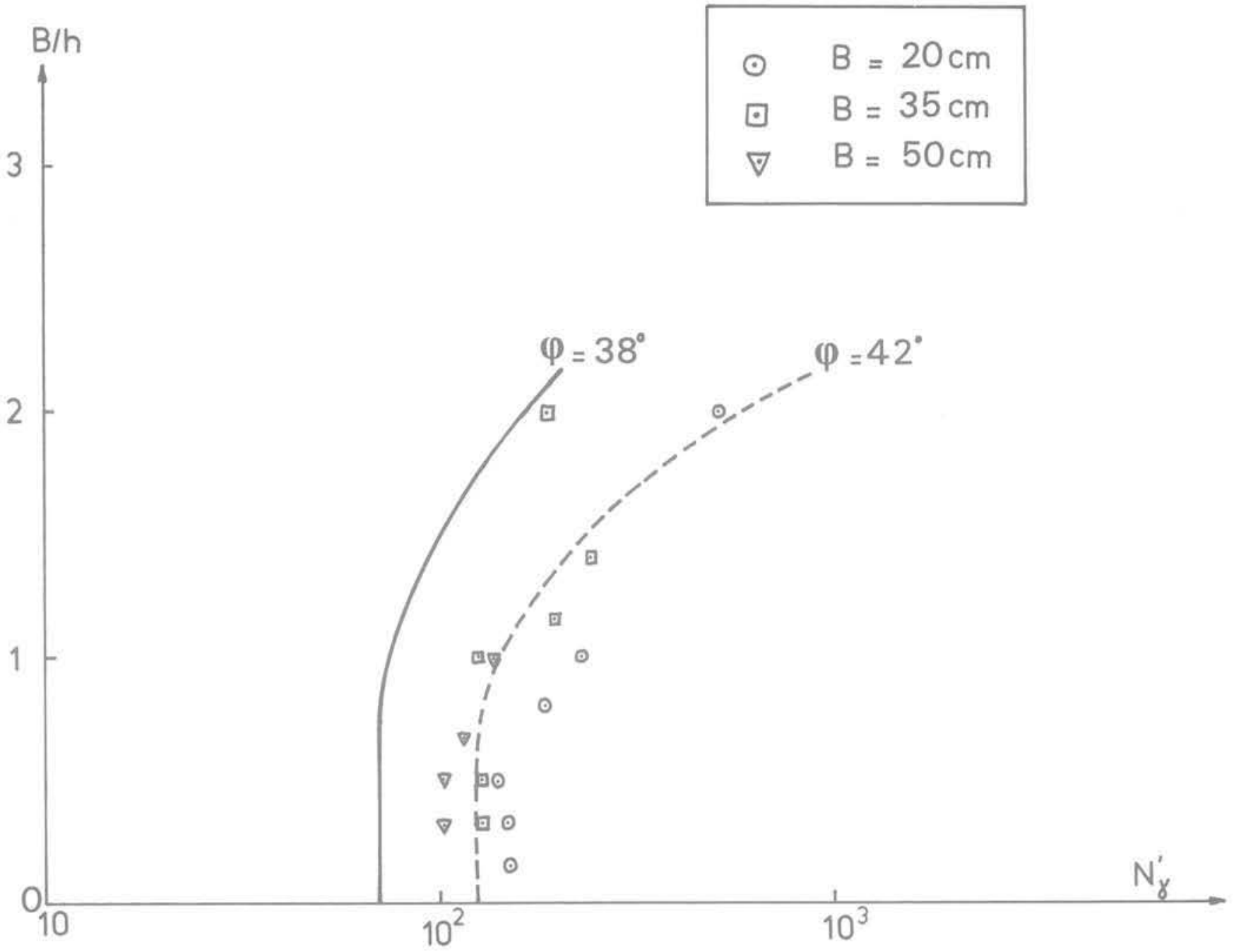

Fig. 40 Comparaison entre résultats théoriques et résultats expérimentaux

\section{Conclusion}

Nous ne reviendrons pas ici sur les aspects théoriques, liés au Calcul à la rupture, contenus dans cet article et nous nous bornerons à mettre en relief les apports du point de vue du calcul pratique :

On a donné la formule permettant, pour une couche de sol d'épaisseur limitée et de cohésion croissant linéairement avec la profondeur, de prendre en compte le gradient de cohésion pour l'évaluation de la capacité portante d'une semelle filante par la méthode de superposition (formule linéaire): ce résultat est basé sur l'équivalence "poids volumique-gradient vertical de cohésion ».

On a donné des formules et des abaques permettant de procéder commodément au calcul exact de cette capacité portante en prenant en compte tous les effets de couplage (calcul global).

L'utilisation de ces abaques « iso- $\mu_{c}$ " met en évidence une correction majoratrice, qui peut être importante, sur les résultats obtenus par la méthode de superposition classiquement utilisée pour le calcul de la capacité portante des semelles filantes sur un sol homogène.

Elle apporte une réponse précise à des problèmes d'usage courant, traités en l'état actuel, de manière empirique, par deux types de méthodes. Le caractère dangereux de l'une de ces méthodes a été mis en évidence. L'autre méthode est surement conservatrice, mais peut rendre la construction très coûteuse et parfois impossible.

Elle permet également de montrer l'effet stabilisateur très marqué, du point de vue de la capacité portante, de l'angle de frottement interne pour les sols peu frottants.

\section{Références Bibliographiques}

BERTHET D., HAYOT J.C., SALENÇON J. (1972) Poinçonnement d'un milieu semi-infini en matériau plastique de Tresca non homogène. Archives of Mech., vol. 24, $n^{\circ} 1$, pp. 127-138

COULOMB C.A. (1773) - Essai sur une application des règles de Maximis et Minimis à quelques problèmes de statique relatifs à l'architecture. Mémoires présentés à l'Académie Royale des Sciences.

COUSSY O. et SALENÇON J. (1979) - Analyse de la stabilité des ouvrages en terre par le calcul à la rupture. Annales des Ponts et Chaussées, $n^{\circ} 12$, pp. 7-35.

DAVIS E.H. et BOOKER J.R. (1971) - The bearing capacity of strip footings from the standpoint of plasticity theory. Research report, Civil Engineering Laboratories, University of Sydney.

DUNCAN J.M. et BUCHIGNANI A.L. (1973) - Failure of underwater slope in San Francisco Bay. Jni Soil Mech. and Foundation Division, ASCE.

GIROUD J.P., TRAN-VÔ-NHIEM et OBIN J.P. (1973) Tables pour le calcul des fondations. Tome 3, Dunod, Paris.

HABIB P. (1958) - La dispersion des résultats des essais de sols. Annales de I'I.T.B.T.P., n 130, pp. 1088-1094.

HABIB P. (1961) - Force portante et déformation des fondations superficielles. Annales de I'I.T.B.T.P., $n^{\circ} 163-164$, pp. 759-772. 
LADE P.V. et DUNCAN J.M. (1973) - Cubical triaxial tests on cohesionless soil. Jnl of Soil Mech., and Foundation Division, vol. 99, $n^{\circ} \mathrm{SM} 10$.

LEONARDS G.A. (1968) - Les fondations. Dunod, Paris. LE TIRANT P. (1976) - Reconnaissance des sols en mer pour l'implantation des ouvrages pétroliers. Editions Technip, Paris.

LUNDGREN $\mathrm{H}$. et MORTENSEN K. (1953) - Determination by the theory of plasticity of the bearing capacity of continuous footings on sand. Proc. 3rd Int. Conf. Soil Mech., Zurich, vol. 1, pp. 409-412.

MANDEL J. et SALENCON J. (1969) - Force portante d'un sol sur une assise rigide. C.R. $7^{\mathrm{e}}$ Cong. Int. Méc. Sols, Mexico, vol. 2, pp. 157-164.

MANDEL J. et SALENCON J. (1972) - Force portante d'un sol sur assise rigide (étude théorique) Géotechnique, vol. $22, n^{\circ} 1$, pp. 79-93.

MATAR M. et SALENCOON J. (1977) - Capacité portante d'une semelle filante sur sol purement cohérent d'épaisseur limitée et de cohésion variable avec la profondeur. Annales de I'I.T.B.T.P., $n^{\circ} 352$, pp. 93-108 - Revue Française de Géotechnique, $n^{\circ} 1, p p, 37-52$.

MATAR M. (1978) - Capacité portante des fondations superficielles. Thèse de Docteur-Ingénieur Génie Civil, E.N.P.C., Déc. 1978, Paris.

OBIN J.P. (1972) - Force portante en déformation plane d'un sol verticalement non homogène. Thèse U.S.M., Grenoble.

OLSZAK W. RYCHLEWSKI J. et URBANOWSKI W. (1962) - Plasticity under non-homogeneous conditions. Advances in Applied Mechanics, Ac. Press, N.Y., pp. $132-214$.
SALENÇON J. (1974) - Théorie de la plasticité pour les applications à la mécanique des sols. Eyrolles, Paris.

SALENCCON J. (1978) - Calcul à la rupture et plasticité. Cours E.N.P.C., Paris.

SALENCCON J., BARBIER M. et BEAUBAT M. (1973) Force portante d'une fondation sur sol non homogène. C.R. $8^{\circ}$ Cong. Int. Méc. Sols, Moscou, vol. 1.3., pp. 219-224

SALENCCON J., FLORENTIN P, et GABRIEL Y. (1976) Capacité portante globale d'une fondation sur un sol nonhomogène. Géotechnique, vol. 26, n², pp. 351-370.

SALENCCON J. et HALPHEN B. (1979) - Calcul des structures inélastiques. chap. I " Le comportement élastoplastique ». Cours E.N.P.C., Paris.

SALENCCON J. et MATAR M. (1979) - Bearing capacity of surface foundations. 3rd ASCE/EMD Specialty Conference, University of Texas, Austin, U.S.A. 17-19 sept. 1979.

SHIELD R.T. (1954) - Plastic potential and Prandtl bearing capacity solution. Jnl Appl. Mech. Trans. ASME, vol. 21. pp. 193-194.

TERZHAGI K. $(1943,1965)$ - Theoretical soil mechanics. John Wiley and Sons.

TOURNIER J.P. (1972) - Comportement d'une couche compressible d'épaisseur limitée par un substratum rigide et soumis à une charge verticale appliquée à une semelle filante. Thèse de Doctorat (Ph.D), Université de Sherbrooke, P.Q. Canada.

TOURNIER J.P. et MILOVIC D.M. (1977) - Etude expérimentale de la capacité portante d'une couche compressible d'épaisseur limitée. Géotechnique, 27, $n^{\circ} 2$, pp. $111-123$. 